KfK 4612

Juli 1989

\title{
A Physical Model of Prebreakdown in the Hollow Cathode Pseudospark Discharge Based on Numerical Simulations
}

K. Mittag Institut für Datenverarbeitung in der Technik 

Kernforschungszentrum Karlsruhe

Institut für Datenverarbeitung in der Technik

KfK 4612

\section{A PHYSICAL MODEL OF PREBREAKDOWN IN THE HOLLOW CATHODE PSEUDOSPARK DISCHARGE BASED ON NUMERICAL SIMULATIONS}

Karl Mittag

Kernforschungszentrum Karlsruhe $\mathrm{GmbH}$, Karlsruhe 
Als Manuskript vervielfältigt

Für diesen Bericht behalten wir uns alle Rechte vor

Kernforschungszentrum Karlsruhe GmbH

Postfach 3640, 7500 Karlsruhe 1

ISSN 0303-4003 


\title{
Zusammenfassung
}

\author{
Ein physikalisches Modell der \\ Anfangsphase einer Hohlkathodenentladung (Pseudofunken) \\ auf der Grundlage von numerischen Simulationen
}

Der Pseudofunke ist eine zylindersymmetrische Gasentladung mit einem Loch in der Kathode und einem Hohlkathodenraum im Außenbereich der Kathode. Es wird ein physikalisches Modell der Anfangsphase des Pseudofunkens vorgestellt, das auf der Analyse von Ergebnissen numerischer Simulationen beruht.

Das Auftreten eines gut fokussierten Elektronenstrahis mit hoher Stromdichte ist das Ergebnis eines erhöhten elektrischen Feldes im Hohlkathodenraum, welches durch eine positive Raumladung hervorgerufen wird. Das beobachtete überexponentielle Wachstum der Dichten als Funktion der Zeit bei dem Loch in Achsennähe wird durch einen positiven Rückkopplungsmechanismus hervorgerufen. Dieser beruht einerseits darauf, daß das elektrische Feld, der lonisationskoeffizient, die Driftgeschwindigkeiten und als Folge davon auch die Dichten der Elektronen, der positiven Ionen und der Raumladung alle ihr Maximum auf der Achse beim Loch haben. Zum anderen läßt sich aus der Kontinuitätsgleichung ableiten, daß die relative Änderung der Elektronendichte gegeben ist durch die Summe eines lonisierungs- und eines Raumladungsterms. Letzterer ist um so größer, je mehr Elektronen an einem Ort vorbeidrifteten und ist daher für das starke überexponentielle Wachstum der Dichten in der Zündphase verantwortlich.

In der vorliegenden Arbeit werden außerdem sowohl die Grundzüge des numerischen Modells beschrieben, als auch über die Ergebnisse umfangreicher Programmtests berichtet. Danach können große numerische Fehler oder Instabilitäten ausgeschlossen werden, so daß die Simulation die physikalischen Vorgänge qualitativ richtig beschreibt.

Das wesentlichste Ergebnis der Simulation, das äußerst schnelle Auftreten eines gut fokussierten, hochintensiven Elektronenstrahls auf der Achse in der Nähe des Loches entspricht experimentellen Beobachtungen. 


\begin{abstract}
A Physical Model of

Prebreakdown in the Hollow Cathode Pseuc'ospark Discharge

Based on Numerical Simulations
\end{abstract}

The pseudospark is a gas discharge in a cylindrical geometry with a hole in the cathode and a hollow cathode region behind it. Based on the analysis of numerical simulations a physical model of the prebreakdown phase of the pseudospark is presented.

The occurence of a well focused high intensity electron beam is a result of an enhanced electric field in the hollow cathode caused by a positive space charge. The observed overexponential growth of densities in time on axis close to the hole is caused by a positive feedback mechanism. On one hand, this is based on the fact that the electric field, the ionization coefficient and the drift velocities, and as a consequence also the electron, the positive ion, and hence also the space charge density all have their maximum on axis at the hole. On the other hand, it follows from the continuity equation that the relative change in electron density at a given point is the sum of an ionization and of a space charge term. The latter one is the larger the more electrons have drifted by, and it is the cause for the rapid overexponential growth of densities in the ignition phase.

Further, in the present work the essentials of, and tests performed on the numerical code are described. These give confidence that large numerical errors or instabilities can be excluded in the simulations, and that indeed the discharge physics is modelled properly, and that the results describe the physics qualitatively correct.

The main feature of these simulations, the extremely rapid appearance of a well focused, high intensity electron beam on axis, originating at the hole, is also seen in experiments. 


\section{Contents}

$\begin{array}{ll}\text { 1. Introduction } & 1\end{array}$

2. Fluid model for the hollow cathode region 2

2.1 The basic equations 2

2.2 The boundary condition at the hole 4

$\begin{array}{ll}2.3 \text { Accuracy tests performed on the numerical code } & 6\end{array}$

$\begin{array}{lrr}\text { 3. Results of the Simulations } & 8\end{array}$

$\begin{array}{ll}3.1 \text { The discharge parameters } & 8\end{array}$

3.2 The dependence of the current density growth rate on $\begin{array}{ll}\text { the primary ionization coefficient } & 8\end{array}$

$\begin{array}{ll}3.3 \text { The buildup of charges in the hollow cathode region } & 10\end{array}$

4. Conclusions 13

$\begin{array}{ll}\text { References } & 15\end{array}$

$\begin{array}{lr}\text { Acknowledgement } & 16\end{array}$ 


\section{Introduction}

The pseudospark discharge is a special form of hollow cathode discharge at pressures around $30 \mathrm{~Pa}$, voltages between 1 and $400 \mathrm{kV}$, and typically 1 to $5 \mathrm{~cm}$ gap between anode and cathode. It promises applications in fast high power switches, in well pinched high intensity pulsed electron beams, as well as in point-like X-ray sources ${ }^{12}{ }^{3}$.

A comprehensive numerical simulation of the physics in pseudospark devices designed for such applications would have to take into account many primary effects on an atomic scale like elastic scattering, exitation, ionization, dissociation of molecules, attachment, detachment, charge exchange, and prompt and delayed emission of photons. In additon, secondary effects at the surfaces like the creation of electrons by photoeffect, or impinging slow and fast ions as well as fast neutral molecules would have to be considered, not forgetting backscattering. The motion of the charged particies would have to regard the externally applied electric and magnetic fields as well as those produced by the charges and currents in the later stages of the discharge. Also, the complex geometrical set-up together with the effect of surface layers on dielectric and metal materials play an important role in real pseudospark diodes.

From experiments it is known that the buildup of the pseudospark discharge is well separated into a slow, low current predischarge, and a very fast, high current main discharge. The former is governed by the motion of charged particles in the electric field together with the buildup of charge carriers mainly in the hollow cathode region. On the other hand, the latter is dominated by the combined action of electric and self-magnetic fields in the previously created plasma. The task to simulate such a complex phenomenon is beyond the possibilities of present-day computers. In this work we limit our model to the most essential features of the prebreakdown phase ${ }^{4}$. The geometry of the model diode can be seen in Fig. 1. It is a single gap diode with a hole at the center of the cathode allowing for a hollow cathode region at the outer side of the cathode.

For the parameter ranges of voltage and pressure at which pseudospark discharges typically are observed, the ratio of the electric field $E$ to the background density $\mathrm{N}, \mathrm{E} / \mathrm{N}$, generally is well above $5 \times 10^{-18} \mathrm{Vm}^{2}$ in the gap region between anode and cathode. At these high $E / N$ - values the charge carriers are continuously accelerated. Their velocity depends on the electric field along their whole path. Since the cross sections for the various types of collisions are strongly velocity dependent, a Monte Carlo type of simulation is appropriate for this region ${ }^{5}$.

On the other hand, in the hollow cathode region the E/N-values typically are below $5 \times 10^{-18} \mathrm{Vm}^{2}$. For this parameter range the charge carriers lose the energy, they pick up in the electric field, again in frequent elastic and inelastic collosions with the neutral background gas molecules. As a result, the charged particles drift along the electric field lines. Their average drift velocity only depends on the value of the local electric field at the position under consideration. For this equilibrium situation a fluid model describing the drift motion is applied in the model presented below.

This model comprises the time dependent continuity equations for ions and electrons, describing where the particles come from, where they drift to, as well as the generation of new ones by ionization in the discharge volume, and of secondaries 
e.g. due to photo effect at the boundaries. In addition, the equations of motion are replaced by the relation between drift velocity and electric field, obtained by anaIytical fits to experimental data. (Thus also the Lorentz force equation is implicitely included in this model, justifying to talk about attraction of electrons by a positive charge, later on in the text). Also, the physical effects determining the source terms in the continuity equations - such as ionization, exitation, secondary electron emission due to photo effect or ion impact at the walls - are input to the model as functions fitted to experimental data. Further, Poisson's equation is solved in the whole interior of the model diode, taking the change of the potential due to space charge into account.

This coupled set of differential equations is solved simultaneously, and iteratively as a function of time in a two-dimensional, rotational symmetric r-z-geometry. A computer program written according to this swarm model for an axially symmetric discharge in a plane parallel plate electrode geometry ${ }^{6}$ founded the basis for our work.

\section{Fluid model for the hollow cathode region}

\subsection{The Basic Equations}

The basic equations comprising this model ${ }^{6}$ are given below for the case of only two fluids, namely electrons (e), and positive ions (p). The program can handle negative ions as well, and even more fluid components could be added, in principle. The equations are solved in a loop in the sequence they are written below, iterating for each time step until the most relevant quantities - potential $\phi$ and electron density $\rho_{e}$ - differ not more than preset values from one iteration to the next. At the start of the simulation an initial electron and positive ion distribution has to be assumed.

Space charge: $\quad \rho=\rho_{p}-\rho_{e}$

Poissons equation: $\Delta \phi=\frac{\rho}{\epsilon_{0}}$

Electric field: $\quad \vec{E}=-\nabla \phi$

Equations of motion: $\begin{aligned} & \vec{w}_{e}=f_{e}(E, N) \vec{E}=\text { electron drift velocity } \\ & \vec{w}_{p}=f_{\rho}(E, N) \vec{E}=\text { positive ion velocity }\end{aligned}$

Swarm paramters: $\quad \alpha=f_{a}(E, N)=\frac{d \rho_{e}}{\rho_{e} d s}$

$a_{e x}=f_{a e x}(E, N)$

Boundary Condition for secondary electrons:

$$
\rho_{e} w_{e}=\gamma_{\rho} \rho_{\rho} w_{\rho}+\Gamma_{\phi} \int_{0}^{g} \int_{0}^{R} g\left(z^{\prime}, r^{\prime}, r\right) \alpha_{e x} \rho_{e} w_{e} r^{\prime} d r^{\prime} d z^{\prime}
$$

Continuity equations: $\frac{\partial \rho_{e}}{\partial t}=\alpha \rho_{e} w_{e}-\nabla\left(\rho_{e} \vec{W}_{e}\right)$

$$
\frac{\partial \rho_{\rho}}{\partial t}=\alpha \rho_{e} w_{e}-\nabla\left(\rho_{\rho} \vec{w}_{\rho}\right)
$$


In these equations $a$ is the first Townsend cefficient describing the relative increase of electron density due to ioniztion by electron impact on a given path length ds along the electric field lines. $a_{e x}$ is similarly defined and yields the number of photons due to exitation by electron impact. $\gamma_{p}$ is the secondary coefficient for electron generation by ions impinging on the surface. $\Gamma_{\phi}$ is a similar coefficient for photo effect. $a_{e x} \rho_{e} W_{e}$ gives the photon flux density at a position $\left(\mathrm{z}^{\prime}, \mathrm{r}^{\prime}\right)$ in the hollow cathode region. $g\left(z^{\prime}, r^{\prime}, r\right)$ relates to the probability that a photon originating at that position reaches a point at the surface. The discharge volume extends to the radius $R$, and the axial extent of the hollow cathode is $\mathrm{g}$. Additional equations, solved simultaneously as well, consider the decrease of the gap voltage due to discharging of an external capacitor by the discharge current in the gap.

The continuity equations are solved for the hollow cathode region only, using a non-equidistant, rectangular mesh of $38 \times 17$ lines in rotational symmetric in $r-z-$ geometry (Fig. 1). The hybrid method of characteristics is used to solve these differential equations, choosing for each fluid a coordinate system which moves along with it $^{6}$. For example, the continuity equation for the positive ions thus is transformed into the following integral equation, which follows the positive ions from the time $t_{1}$ at the location $\left(z_{1}, r_{1}\right)$ to the time $t_{2}$ at the location $\left(z_{2}, r_{2}\right)$ :

$\rho_{\rho}\left(t_{2}\right)=\rho_{\rho}\left(t_{1}\right) \exp \left(-\int_{t_{1}}^{t_{2}} \nabla \vec{w}_{\rho} d t\right)+\int_{t_{1}}^{t_{2}} \alpha \rho_{\mathrm{e}} w_{e} \exp \left(-\int_{t}^{t_{2}} \nabla \vec{w}_{\rho}\left(t^{\prime}\right) d t^{\prime}\right) d t$

The divergence terms describe deviations from the behavior of an incompressible fluid, e.g. the attraction or repulsion by the Coulomb force, or a non-linear dependence of the drift velocity on the electric field. $a \rho_{e} w_{e} d t$ gives the increase of ion density due to ionization.

Numerically the calculation of $\nabla \vec{w}_{e}$ and $\nabla \vec{w}_{\rho}$ - both functions of the electric field requires special care to minimize errors. As the derivatives of the electric field components vary rapidly close to the hole, the problem was transformed by making use of Maxwell's equation

$\nabla \vec{E}=\rho / \epsilon_{0}$

and

$\nabla \vec{w}=\nabla(f(E, N) \vec{E})=\frac{d f}{d E} \nabla E \cdot \vec{E}+f \frac{\rho}{\epsilon_{0}}$

$d f / d E$ is calculated analytically from the given function $f(E)$. The numerical calculation of $\nabla E$ remains. However, since the magnitude of $\vec{E}$, $E$, varies much more smoothly across the hollow cathode region than the electric field components, the accuracy has been improved considerably by this transformation.

In order to be economic both in computing time and storage the mesh was chosen finest close to the cathode hole, where the electric field and the ionization are largest. From here the mesh spacing gradually increases towards the outer radial and axial boundaries.

Poisson's equation is solved in all of the interior of the geometry shown in Fig. 1 by means of finite difference methods using boundary fitted coordinates ${ }^{8}$. Thus the 
electric field derived from the potential takes into account the distortion caused by the hole in the cathode, as well as the field changes due to space charge effects.

Further details about the numerical methods are given elsewhere ${ }^{56}$.

\subsection{The Boundary Condition at the Hole}

A principle problem arises from the fact that the physics in the hollow cathode region and in the gap between anode and cathode is quite different. The $\mathrm{E} / \mathrm{N}$-values under consideration in this study are chosen such that in the hollow cathode region just no run-away conditions occur for the electrons, and the fluid model can be applied. As a consequence $\mathrm{E} / \mathrm{N}$ is chosen to be about $4 \times 10^{-18} \mathrm{Vm} \mathrm{m}^{2}$ on axis at the hole boundary. Inside the hole and in the diode gap $E / N$ is much higher than this value, and the continuous acceleration of the electrons could be treated by two-dimensional Monte-Carlo methods. A major problem would be to couple these completely different simulation methods together at the hole boundary. At present this has not been achieved.

Physically these two regions are coupled by particle and photon fluxes crossing the boundary. For the treatment of the hollow cathode region the photon flux coming from the gap is neglected, and the positive ion flux is estimated from the following arguments.

The positive ion flux has to be continuous at the boundary. Then, assuming initially at the start of the simulation a uniform positive ion density in all of the hollow cathode region including on the hole boundary, this density had to remain constant in time, if no additional sources for positive ions were included, and space charge influence could still be neglected. The reason for this is that the positive ions move like an incompressible fluid, since their drift velocity taken from fits to experimental data is proportional to the electric field.

However, new positive ions are created by primary ionization by electrons. If these new-born ions are added to the original ones a density gradient builds up, because ionization is the larger the higher the electric field is. The positive ion density then increases from the hollow cathode walls towards the hole. This density gradient at the hole boundary relates to an additional ion flux entering the hole. In previous publications ${ }^{4}{ }^{5}$ on this subject the positive ion flux at the hole boundary was estimated by extrapolating the ion density into the hole region, adding ions created by ionizing electrons leaving the hole as well. By this trick the simulation inside the hollow cathode region can be performed without any additional parameter depending on the physics in the diode gap. An obvious shortcoming of this approach is that once the density gradient at the hole boundary is established it will cause the positive ion flux to continue to increase in time due to a purely numerical feed back mechanism, even if the electron flux leaving the hole would be constant in time or die out. Thus, most probably this boundary model (1) overestimates the positive ion flux coming from the gap at the final stages of the simulation.

In model (2) this problem is avoided by neglecting that part of the incoming ion flux which is driven by the density gradient at the boundary. Instead it is assumed that at each time step the density inside the hole equals the density at the boundary. Otherwise model (2) is identical to model (1). Proceeding in this manner the continuity at the boundary is insured. However, the positive ion flux entering from 
the gap most probably is underestimated for a discharge which builds up in time, because the electric field increases towards the gap, and therefore also the ionization. From this follows that in reality more ions will be created in the hole than at the boundary. The real physical solution will lie between model (1) and model (2). In case of an electron density decreasing in time model (2) yields a positive ion density constant in time.

A third possibility to tackle this problem is to introduce a parameter which sums up the physics inside the diode gap (model (3)). Such a parameter can be based on the idea that the flux of positive ions entering the hollow cathode region through the hole certainly is related to the electron flux leaving the hole, for example, because the ionization by electrons is proportional to the electron flux. Such an assumption is supported by time dependent one-dimensional MonteCarlo simulations for pre-breakdown of a discharge in a plane parallel plate geometry ${ }^{9}$ which showed that the positive ion flux at the cathode is roughly an order of magnitude less then the electron flux at the anode. Exept for transients at the beginning of this simulation the ratio of these two fluxes turned out to be constant in time. An obvious disadvantage of model (3) is that it creates an unreal discontinuity at the boundary, because inside the hollow cathode region ions are created by ionization. Another shortcoming is a too fast response in ion density when the electron density is changing, neglecting that the ion drift velocity is two orders of magnitude smaller than the electron drift velocity.

These three models were compared for the following discharge parameters : gap voltage $70 \mathrm{kV}$, pressure $470 \mathrm{~Pa}$ (3.5 Torr), swarm parameters as given by ${ }^{10}$, photon secondary coeffiecient 0.02 , no secondary electrons due to ions. As a result the current density at the hole boundary is shown as function of radius and time for these three models in Fig. 2 to 4 . It is seen that in all three cases the qualitative growth behavior is the same. After the input electron distribution has left the hollow cathode area the current density grows exponentially in time. The growth is identical for the different models as long as the positive space charge in the vicinity of the axis does not change the electric field noticable. Once this happens the current density grows overexponentially, and an extremely well focused electron beam is formed around the axis.

The time required for the onset of a pseudospark to develop is 102 ns for model (1), 133 ns for model (2), and 174 ns for model (3). Depending on the different incoming ion fluxes it takes more or less time for a critical positive space charge to be reached (note the different time scales in the figures).

For the parameters chosen model (3) definitely underestimates the ion flux, because the ion density created by ionization inside the hollow cathode turned out to be appreciably larger than that resulting from the incoming flux. As a consequence, for model (3) the ion density at a fixed time even decreases towards the hole in the vicinity of the boundary, which certainly is unreal in this region of the discharge. Of course this shortcoming could be overcome by enlarging the free parameter describing the ratio of ion to electron flux at the boundary until the discontinuity disappears. This approach leads to model (2), however.

It is noticed that models (1) and (2) give rather similar results. This is explained by the fact that the ion density is caused mainly by ionization in the hollow cathode and hole regions, and not by ions coming from the gap, as the slow ions on axis move only $1.5 \mathrm{~mm}$ from the hole boundary inside the hollow cathode during $100 \mathrm{~ns}$. As long as the simulation takes into account properly the growth of ion 
density caused by ionization, additional incoming slow ion flux is of less relevance. These arguments lead to the choice of model (2) as a standard for further simulations, and give confidence that the results presented below are qualitatively correct, even though the discharge physics in the gap region is neglected.

Another problem at the hole boundary is related to the circumstance that the electric field is calculated in all of the discharge region, whereas the space charge term entering Poisson's equation is only known inside the hollow cathode region. In order to avoid numerical difficulties arising from a discontinuity in space charge density at this boundary, the space charge density is extrapolated into the hole region, decreasing exponentially in distance, typically a factor of $10^{12}$ across the disk. Changing this decay factor by orders of magnitude had only a minor effect on the results. This extrapolation is based on the fact that because the electric field is increasing towards the anode, also the particle velocities are increasing, hence there densities are decreasing, assuming a constant particle flux density in this argument.

\subsection{Accuracy Tests Performed on the Numerical Code}

An integral accuracy test of the electric field solver for the hollow cathode region can be based on the idea that for zero space charge the divergence of the electric field vanishes. Hence, making use of Gauss' theorem, the total electric flux entering the hollow cathode region must be equal to the total electric flux reaching the walls inside this region. A numerical integration of the two related surface integrals yielded that the flux entering the hole agrees with that reaching the walls to within $2 \%$.

The next accuracy test was concerned with the time dependent motion of the electrons from the metal walls inside the hollow cathode region towards the hole. It is well known that the method of characteristics tends to smear out steep gradients, much more so than the more recently developed flux corrected transport methods. In order to quantify this deficiency the following numerical experiment was set up.

Starting from a uniform electron density at the start of the simulation the electron density at the wall opposite to the hole was increased instantaneously by a factor of 2 for radii less than $5 \mathrm{~mm}$. The electron density on the remainder walls was unchanged. This electron density distribution at the walls was then kept constant in time, and the electron pulse leaving the wall opposite to the hole was followed as it moved from the wall towards the hole.

For this calculation the electric field was kept constant in time, hence neglecting the space charge source term. Further the primary ionization by electron collisions was set to zero, and the electron drift velocity was approximated by a function exactly proportional to the electric field. From these simplifications follows that the divergence of the electron drift velocity is zero, the electrons move like an incompressible fluid, their density remains unchanged as they drift from the walls towards the hole (the increase in flux density reflects itself as an increase in the drift velocity alone). This allows an easy way to calculate the exact time independent solution of this test run.

On axis the time required for the electrons to traverse the hollow cathode region can be calculated straight forward by numerical integration to be 12.4 ns for a anode-cathode gap voltage of $20 \mathrm{kV}$ and a background nitrogen gas pressure of 
$133 \mathrm{~Pa}$. At this time the front of the electron pulses reaches the hollow cathode boundary on axis, whereas for larger radii this front is still inside the hollow cathode region, because the drift velocity is smaller off axis than on axis, also the drift path length is longer off axis. The following plots are three-dimensional for the hollow cathode region only. The origin of the $r$-z-coordinate system lies on the diode axis next to the hole (see Fig. 1). In Fig. 5 the broadening of the pulse due to deficiencies in the method of characteristics can clearly be seen. The pulse maximum on axis at the hole is $22 \%$ too small compared to the theoretical solution.

The simulation was continued until a stationary solution was reached (Fig. 6). On axis at the hole the numerical solution now agrees exactly with the theoretical one, whereas off axis the pulse boadening is still appreciable. As is shown below, across the hole boundary the edge of the pulse theoretically is at $r=0.88 \mathrm{~mm}$, where the pulse heigth is $28 \%$ too small. The electron flux leaving the hole for radii less than $r=0.88 \mathrm{~mm}$ is $19 \%$ less than the corresponding flux leaving the opposite wall for radii less than $5 \mathrm{~mm}$. The electron flux up to a radius of $0.97 \mathrm{~mm}$ across the hole has to be included in order that these source and sink fluxes are identical. This corresponds to a pulse broadening in radius at the hole of $10 \%$, and in area of $22 \%$. However, the total electron flux leaving the hole agrees to $0.6 \%$ with the total flux leaving the metal walls inside the hollow cathode region.

In a similar manner the numerical solution was tested with respect to the positive ion movement. For this case a step pulse of ions was entered the hollow cathode region at the hole boundary for radii less than $0.88 \mathrm{~mm}$. The edge of this pulse should reach the opposing wall at a radius of $5 \mathrm{~mm}$. Fig. 7 shows the result after 1 ion passage on axis (1380 ns), when the pulse is still $27 \%$ to small on axis. Fig. 8 gives the answer at 2.9 ion passages on axis (4000 ns). It is seen that instead of reaching the opposite wall some ions are moving backwards towards the cathode back wall. They have diffused out of the pulse to larger radii into comparitively higher electric field, where they move faster than ions at the pulse edge. The pulse was not followed for longer times because of the exessive computation time required, and because the pseudospark behaviour develops much more rapidly.

Summing up this analysis, it is concluded that steep density gradients tend to be smeared out when the electrons or ions are traversing the hollow cathode region, the density at a sharp ridge is reduced typically by 20 to $30 \%$ compared to the theoretical solution. The flux diffusing out of the pulse amounts to about $20 \%$, but the total flux is conserved perfectly to better than $1 \%$. For the purpose of yielding a qualitative understanding of the prebreakdown phenomena in a pseudospark the accuracy is adequate, however. This the more so, because in the simulations to be reported an extremely localized high density plasma builds up in the vicinity of the hole, and any numerical errors related to the method of characteristics would just work in the opposite direction to smear out this localized density.

A further test was to check on the exponential growth in electron density resulting from primary ionization when the electrons pass through the hollow cathode region. This can easily be checked only on axis, because there the electron motion is one-dimensional. For this numerical experiment the parameters were left as above, only turning on the primary ionization in addition. Evaluating the integral over the primary ionization coefficient along the axis, a growth factor of 5.98 is obtained. The method of characteristics yields a growth factor of 5.43 , or $9 \%$ to small at $12.4 \mathrm{~ns}$, when the pulse has just reached the hole. A growth factor of 7.00 , or $17 \%$ to high is obtained for the stationary solution. The reason for this overes- 
timate in growth lies in the four-point linear two-dimensional interpolation which is used in order to estimate the ionization coefficient between mesh points. A higher order interpolation formula would reduce this error, which seems to be tolerable for a qualitative analysis, however.

The scaling of the discharge behavior with voltage and pressure is another point of interest. The primary coefficients for ionization and photon exitation scale proportional to $N \cdot f(E / N)$. The drift velocities are proportional to $E / N$. Thus reducing $E$ and $N$ by the same factor while increasing these primary coefficients by the same factor should leave the discharge buildup unchanged. This was verified by comparing a run at $70 \mathrm{kV}, 470 \mathrm{~Pa}$ with one at $20 \mathrm{kV}$ and $133 \mathrm{~Pa}$.

Another test of the computer code was done by comparing the results obtained after 100 time steps of 0.1 ns each with those obtained after 1000 time steps of 0.01 ns each. The densities agreed within $1 \%$ for these two cases.

Also, it was varified that chosing a factor of 2 denser mesh did not change the results to more than $10 \%$ in the significant quantities.

\section{Results of the simulations}

\subsection{The Discharge Parameters}

The parameters of the simulations to be described are close to the experimental conditions under which pseudosparks are observed:

$\begin{array}{ll}\text { outer radius of the discharge chamber } & =10.0 \mathrm{~mm} \\ \text { gap between anode and cathode } & =10.0 \mathrm{~mm} \\ \text { disk thickness } & =3.0 \mathrm{~mm} \\ \text { radius of the hole in the cathode } & =2.5 \mathrm{~mm} \\ \text { length of hollow cathode region } & =5.0 \mathrm{~mm} \\ \text { voltage between anode and cathode } & =20 \mathrm{kV} \\ \text { background gas pressure } & =133 \mathrm{~Pa} \text { (1 Torr) } \\ \text { primary coefficients } & \\ \text { secondary coefficient for photo effect } & =0.02 \\ \text { secondary coefficient for ion impact } & =0\end{array}$

The geometry, the numerical mesh chosen (in radial direction 38 lines, in axial direction 17 lines in the hollow cathode, 29 lines in the disk, and 27 lines in the gap), and equipotential lines are shown in Fig. 1.

\subsection{The Dependence of the Current Density Growth Rate on the Primary Ioniza- tion Coefficient}

The current density on axis on the hollow cathode boundary is presented in Fig. 9. The parameters varied are the primary coefficients for ionization and photo excitation, which are multiplied by the constant factor ALC3 as compared to the fits of these coefficients to experimental data ${ }^{10}$.

It is seen that if the primary coefficients are taken to be equal to the experimental data $(A L C 3=1)$ no pseudospark should occur according to the simulation, since 
the discharge dies out, the current density falls exponentially in time. This contradicts experimental results which show the occurence of the pseudospark at even lower pressure, corresponding to even smaller primary coefficients. The conclusion is that not all the effects relevant for ionization growth have been included in the model as yet.

Especially the influence of fast ions entering from the gap region and producing secondary electrons at the hollow cathode walls probably has to be taken into account. In order to get an idea how large the additional fast ion flux has to be to contribute significantly to to buildup of the discharge, the following model was tested. As a free parameter it was assumed that for every electron leaving the hole 0.1 electrons are created instantaneously by fast ion impact on the wall opposite the hole inside the hollow cathode at the same radial position. Thus the ion transit time and their radial movement were neglected. The magnitude of this free parameter can be justified by the results of the Monte Carlo simulations quoted above ${ }^{9}$, and by a secondary coefficient for ion impact of 1 , which is the typical order of magnitude for $20 \mathrm{kV}$ nitrogen ions. Under these conditions a buildup of the discharge already was found for ALC3 $=1$, and it developed similarly as in the simulations without fast ions.

Further, the ionization by the collisions of metastable ions with the background gas molecules might be of importance in this context ${ }^{10}$. On the other hand, the contribution of slow ions produced by ionization in the discharge volume to the production of secondary electrons on the hollow cathode walls by ion impact will be small. The reason for this is the low ion velocity. During the predischarge time of typically several $100 \mathrm{~ns}$ the slow ions scarcely move, since it takes the fastest slow ions 1380 ns to transverse the hollow cathode region on axis, and most slow ions are born in the vicinity of the hole. So most slow ions will not have reached the walls before onset of the pseudospark.

As a first step to account for these other effects the primary ionization coefficients were multiplied by the free parameter ALC3 $=3.0$ and 3.5 respectively. A nearly stable discharge is obtained in the first case, whereas a faster growth is seen in the latter one.

The effect of the magnitude of the initial ionization can be studied by comparing the two results given in Fig. 9 for $\mathrm{ALC} 3=3.5$. The initial density, uniform throughout the hollow cathode region, differs by a factor of $10^{4}$ between these cases. After about $10 \mathrm{~ns}$, which corresponds to the time which the electrons close to the axis need to pass the hollow cathode, the arbitrary initial electron distribution has been transformed into the physical solution. Thereafter the current density growths purely exponentially - as is predicted by the analytical solution of the electron continuity equation -, as long as space charge does not enhance the electric field appreciably. Once this occurs the current density growths overexponentially in time. For the case of the higher initial density this happens much earlier. Performing a transformation of the time coordinate on this curve it is seen that the overexponential growth behavior is identical for these two cases. Thus it is proven that the results are independent of the magnitude of the initial uniform distribution, which effects only the time required for the space charge to build up, and hence for the overexponential growth (pseudospark) to start. 


\subsection{The Buildup of Charges in the Hollow Cathode Region}

For the parameters chosen the electrons and slow ions are in equilibrium with the electric field, they move along the field lines, and their local velocity is determined by the local electric field only, which is shown in Fig. 10 to 12 for zero space charge at the start (time $T=0$ ), and, for comparison, in Fig. 13 to 15 at the end of the simulation. The electric field magnitude grows from the walls towards the hole. A similar functional dependence holds for all field dependent functions, like drift velocities and primary ionization coefficient, therefore. The electric field extracts all electrons which are generated inside the hollow cathode through the hole into the gap region. Note that the axial electric field component changes sign inside the hollow cathode region.

On their way from the walls towards the hole the electrons are focused by the radial electric field to a considerable extent towards the axis. For zero space charge, and for the case that the electron drift velocity is exactly proportional to the electric field the motion of the electrons from the walls towards the hole can be traced by applying Gauss' theorem. As an example, it turns out that electrons leaving the wall opposite to the disk inside the hollow cathode region at a radius of $5 \mathrm{~mm}$ are leaving the hole at a radius of $0.88 \mathrm{~mm}$. On axis the electron current density increases from the wall towards the hole boundary by a factor of 14 due to these purely geometrical effects alone. This increase manifests itself nearly completely as an increase in electron velocity. The electron density remains nearly constant, as the functional relation between drift velocity and electric field is close to linear, and hence the electrons - like the ions - nearly move as an incompressible fluid.

The electrons coming from the cylindric part of the wall move maximum $0.7 \mathrm{~mm}$ in $100 \mathrm{~ns}$ in radial direction. Hence they play a negligible role in the hollow cathode predischarge. Electrons which are leaving the walls for radii less than $5 \mathrm{~mm}$ need less than 160 ns to reach the hole, hence the secondary emission from the walls opposite the hole and from the backside of the cathode are the most important ones with respect to the buildup of the discharge. On axis at the hole the electrons have a velocity of $3.2 \mathrm{~mm} / \mathrm{ns}$, that of the ions is $2.8 \mathrm{~mm} / 100 \mathrm{~ns}$; on axis at the hollow cathode wall these velocities are $2.2 \mathrm{~mm} / 10 \mathrm{~ns}$ and $1.7 \mathrm{~mm} / 1000 \mathrm{~ns}$, respectively.

The buildup of electron and positive ion charges inside the hollow cathode is shown in Fig. 16 to 27 at various time steps for the case with ALC3 $=3.5$ and an initial uniform density of 0.1 particle $/ \mathrm{cm}^{3}$ at the start of the simulation time $T=0$. Contributions by ion impact on the walls were not taken into account. 100 ns after the start of the simulation (Fig. 16, 17) the arbitrary initial filling has transformed into the physical solution for the electrons. In comparison, the initial positive ions scarcely move, especially so far away from the axis. Their density gets enhanced by ions newly born by ionization. This ion density increase is least at the cylindric wall where the electric field is smallest, and gets larger towards the axis and towards the hole, having its maximum on axis at the hole. Next to the cylindric wall both electrons and ions get more or less stuck during all of the simulation time, because of the extremely small drift velocities in this region.

From Fig. 9 it is seen that at 400 ns the current density at the hole still grows purely exponentially in time, since the space charge does not enhance the electric field as yet. At this time, the positive ion density at the hole is about three orders of magnitude larger than the electron density, because the electrons are drawn 
out of the hole rapidly, leaving the positive ions nearly stationary behind (Fig. 18, 19).

At later times (Fig. 20 to 27 ) the density peak appearing extremely rapidly during a few ns in the vicinity of the axis close to the hole is the most surprising feature of these simulations. Electron, positive ion and space charge density all grow continously as a function of time in the whole hollow cathode region during all phases of the simulation (apart from the very early stages mentioned above).

The effect of the positive space charge on the electric field at the end of the simulation is shown in Fig. 13 to 15, and for a magnified section at the hole it is given in Fig. 28 to 31 . The radial electric field is increased drastically by up to a factor of 14 in the vicinity of the point-like positive charge $0.01 \mathrm{~mm}$ off axis, amounting already to $30 \%$ of its maximum value. At the radial electric field maximum, 1.9 $\mathrm{mm}$ off axis, this increase remains a less noticable $8 \%$. A substantial increase in radial focussing of the electrons close to the axis at the hole is the consequence. Of course the electrons still follow the field lines, which have changed due to the space charge action.

The total field is reduced close to the hole and enhanced further inside the hollow cathode region as compared to the zero space charge field at $T=0$, as a result of the cumulative action of all the positive charges in the hollow cathode region. This field change amounts to a reduction of $14 \%$ on axis at the hollow cathode boundary, and to a maximum increase of $30 \%$ in the interior. Its effect on the ionization $a$ is less at the hole, where $E / N$ is very high, but larger in the interior of the hollow cathode - resulting at the maximum to a factor of 2 increase at the hollow cathode wall opposite the hole -, because $a$ varies with $E$ as exp(-const/E).

The densities increase exponentially in time as long as the expression determining the growth due to ionization alone,

growth factor $=\int a w_{e} d t=\int a d s$

taken over the electron path, remains constant, and space charge influence can still be neglected. Once this happens, the growth factor increases in time, and the growth becomes overexponential. On axis, from the wall towards the hole the electron density growth caused by ionization amounts to a factor of 524 at $T=0$, but to 1750 at the late state of the simulation. The product $a$ times electron drift velocity $w_{\mathrm{e}}$ which determines the relative change in electron density due to ionization in a given time interval is shown in Fig. 32 to 34 , plotted over a magnified $r$-z-region close to the hole. It is seen that this function varies smoothly in both $r$ and $z$, also at the final state of the simulation. Also the radial dependence of the growth factor is rather smooth, both with and without space charge. On axis it is only $1 \%$ larger than $0.1 \mathrm{~mm}$ off axis.

On the other hand, the electron densities differ by several orders of magnitude across this smali radial distance at the end of the simulation. Analyzing the numerical results, it is found that the reason for the occurrence of the singularity on axis at the hollow cathode boundary is the action of the positive space charge, changing the electric field and thereby attracting electrons rapidly. (Note that the statements that the electrons are attracted by the positive charge or that they follow the field lines changed by this charge are both valid, since charge and field are equivalent in Maxwell's equations). During the last phase of the simulation the growth in electron density due to this effect is much larger than that caused by 
ionization, which can be seen by comparing Fig. 34 and 35 . The drift term $\nabla \vec{w}_{e}$ is over a factor of 10 larger than the ionization term $a w_{e}$ at this location. The combined effect of ionization and drift caused by space charge attraction is shown in Fig. 36. The ionization term dominates inside the volume, whereas the divergence term produces the singularity on axis at the hole.

Summarizing, the appearance of the singularity has its origin in the fact that the electric field, the ionization coefficient and the drift velocities all have their maximum on axis at the hole. Hence the rate at which the charge carriers are multiplied is maximum here, too, and their exponential growth on axis is larger then off axis, which can be verified by analyzing Fig. 2 to 4 . Consequently, the maxima of electron, ion and space charge density are positioned also here. The space charge changes the electric field here most, and its attractive action on the electrons becomes here noticable first, and also has its maximum here. Also the number of electrons attracted by the space charge is largest here, since the electron density maximum is located here, too. These attracted electrons also ionize, and increase the positive space charge at the maximum even further, more than elsewhere. The fact that the positive space charge itself was created by electrons passing by and ionizing at previous times, causes a positive feedback mechanism, and an overexponential growth in electron density is the consequence.

This argument can be made more sound by an approximative solution of the electron continuity equation as follows. If in first approximation a linear relation between electron drift velocity and electric field is assumed, then

$$
\begin{aligned}
\nabla \vec{w}_{e} & \approx \text { const } \nabla \vec{E}=\text { const } \rho=\operatorname{const}\left(\rho_{\rho}-\rho_{e}\right) \\
\rho_{e}\left(t_{2}\right) & =\rho_{e}\left(t_{1}\right) \exp \left(\int_{t_{1}}^{t_{2}}\left(a w_{e}-\nabla \vec{w}_{e}\right) d t\right) \\
& \approx \rho_{e}\left(t_{1}\right) \exp \left(\int_{t_{1}}^{t_{2}} a w_{e} d t\right) \exp \left(\int_{t_{1}}^{t_{2}} \operatorname{const}\left(\rho_{\rho}-\rho_{e}\right) d t\right)
\end{aligned}
$$

(The integral is taken along a trajectory moving along with the electron fluid. $\rho_{e}$ is the electron density at the start of the trajectory at time $t_{3}$ ). If the motion of the ions is neglected, the ion density at a given time $t$ after the start of the discharge can be approximated by

$\rho_{\rho} \approx \int_{0}^{t} a_{e} \rho_{\mathrm{e}} w_{e} d t^{\prime}$

and it results

$\rho_{\mathrm{e}}\left(t_{2}\right) \propto \exp \left(\right.$ const $\left.\int_{t_{1}}^{t_{2}} \int_{0}^{t}\left(a_{\mathrm{e}} \rho_{\mathrm{e}} w_{\mathrm{e}} d t^{\prime}-\rho_{\mathrm{e}}\right) d t\right)$

At the time when space charge starts to change the behavior of the discharge (Fig. 20 to 25) the positive charge is orders of magnitude larger than the negative one, in the vicinity of the hole close to the axis. It follows that the growith in electron density in this region depends in a complicated way exponentially on the time increasing electron density itself, which causes the overexponential growth. This happens first at the location where $\rho_{\rho}$ has its maximum. 
The positive space charge is not fully neutralized by the attracted electrons, because the latter are rapidly extracted out of the hollow cathode region. Because of their over a factor of 100 smaller mobility the ions are left behind, staying where they were created during the extremely rapid predischarge. At the time when the simulation was stopped, the positive ion density was $1.830 \times 10^{13} \mathrm{~cm}^{-3}$, and the electron density was $1.808 \times 10^{13} \mathrm{~cm}^{-3}$ on axis at the hole. It follows that the positive charge is compensated by the electrons to with about $1 \%$.

The electron drift velocity at this location corresponds to an electron energy of 22 eV. Setting this energy - for the sake of curiosity - equal to an electron temperature, the Debye length (which gives the distance over which a charge or electric field is shielded in a plasma) turns out to be $0.008 \mathrm{~mm}$. In the code the smallest mesh size chosen at this point was $0.01 \mathrm{~mm}$ in radial, and $0.12 \mathrm{~mm}$ in axial direction. It is surprising - and may be accidental - that the space charge shielding calculated by the code, and that calculated by a completely different method, based on the Debye length, turn out to be close together in value. The reason for this might be found in the fact that the electron drift velocity determines the speed at which the positive charge can be neutralized by the electrons, and that the thermal velocity plays a similar role in a plasma.

A temperature is neglected in our model - but could be introduced via a diffusion term, in principle. It is reminded that the numerical diffusion inherent in the method of characteristics plays this role in the simulation in a non-controllable manner.

\section{Conclusions}

The multi-fluid model to numerically simulate the prebreakdown of the pseudospark in the hollow cathode region describes the physics qualitatively correct. The method of characteristics chosen to solve the continuity equations tends to a pulse broadening of typically $20 \%$ for a single electron or ion passage through the hollow cathode region.

The model to estimate the flux of slow positive ions entering the hollow cathode region from the main discharge gap is based on extrapolating the positive ion density from the hollow cathode towards the hole. This model is adequate for a qualitative understanding of the underlying physics, since the buildup of a positive space charge in the hollow cathode is mainly due to ionization by electron impact and not by an incoming positive ion flux from the hole region.

Thus the occurence of a well focused high intensity electron beam on axis originating in the hollow cathode at the hole - interpreted as the onset of the pseudospark - certainly is not related to numerical errors or instabilities but corresponds to similar observations in real experiments ${ }^{11} 12$.

The physical picture of the prebreakdown phase of the pseudospark based on the results of the numerical simulations is summarized as follows:

Due to the special hollow cathode geometry with a hole in the cathode the electric field is extremely inhomogenous in the hollow cathode region. The field increases from the walls towards the hole with a radial maximum on axis. A similar space dependence follows for all field dependent quantities, like electron and ion drift velocities, as well as primary ionization and photon emission due to collisions of 
electrons with the background gas molecules. The electrons are focused to a considerable extent towards the axis by the radial electric field.

As long as the space charge does not change the electric field the initial charge densities grow or fall exponentially in time, depending on whether the electrons being extracted out of the hollow cathode through the hole towards the anode can be replenished by electrons newly created in primary or secondary collisions, like ionization by electrons and photo effect at the walls. This exponential growth follows directly from the electron continuity equation, because the change of electron density in time is proportional to the electron density itself. During these initial stages of the discharge all quantities vary smoothly both in axial and radial directions. As a consequence of the electric field maximum on axis the electron, the ion, and the space charge densities also have a maximum on axis, and increase towards the hole.

Since the electrons move over a factor of 100 faster than the ions, they are extracted out of the hollow cathode region rapidly. The positive ions are left nearly stationary behind. Their density grows predominantly due to ions newly born by ionization, and at first gets several orders of magnitude larger than the electron density in the course of the predischarge. At some time this positive space charge starts to shield off the electric field entering through the hole from the anode. Because of this, the electric field is decreased close to the hole and is increased in the interior of the hollow cathode region as compared to the zero space charge case. The influence of the space charge on the spatial dependence of the primary coefficients and the drift velocities is similar, as these quantities all increase with the electric field, all of them still vary smoothly in space. The growth factor for the electrons due to ionization increases because of this change in the electric field, and consequently the particle densities grow overexponentially in time.

The most important efrect of the positive space charge in changing the electric field is the attraction of electrons by the Coulomb force, however. First, this causes an even stronger focussing of electrons towards the axis. They are extracted through the hole, however, so that they do not succeed in fully neutralize the positive charge.

Second, the pseudospark singularity with an overexponential density growth in time on axis at the hole is triggered by the following positive physical feedback mechanism. The relative rate at which the electron density increases close to the hole is mainly determined by the attraction of electrons due to space charge, which is largest on axis at the hole where the positive space charge has its maximum. Also the number of attracted electrons is largest here, since the electron density maximum is located here, too. These attracted electrons also ionize, and increase the positive space charge at the maximum even further, more than elsewhere. As this positive space charge itself was created by electrons passing by and ionizing at previous times, an overexponential growth in electron density, and consequently also in ion density, occurs.

It results an extremely well focused high intensity, but still low current electron beam on axis - interpreted as the onset of the pseudospark - which is extracted out of the hollow cathode region towards the anode.

These predictions agree qualitatively with corresponding experimental results obtained with a streak camera11, or with more sophisticated optical spectroscopy ${ }^{12}$. In order to achieve a more quantitative agreement between sim- 
ulations and experiments the contribution of fast ions entering the hollow cathode from the anode - cathode region, as well as the ionization by metastable background molecules ${ }^{10}$ probably have to be incorporated into the numerical model.

\section{References}

1. K. Frank, J. Christiansen, O. Almen, E. Boggasch, A. Görtler, W. Hartmann, C. Kozlik, A. Tinschmann, G. F. Kirkman, A $40 \mathrm{kV} / 20 \mathrm{kA}$ Pseudospark Switch for Laser Applications, Proc. of the Int. Soc. for Optical Engineering, Innovative Science and Technology Symposium, Los Angeles, 173 (1988)

2. W. Bauer, H. Ehrler, A. Rogner, C. Schultheiss, Review of Effects of the High Power Pseudospark, Proc. of the IX Int. Conf. on Gas Discharges and their Applications, Venice, Italy, 677 (1988)

3. P. Billaut, H. Riege, M. van Gulik, E. Boggasch, K. Frank, R. Seeböck, Pseudospark Switches, CERN-Report 87-13 (1987)

4. K. Mittag, Numerical Simulation of Prebreakdown in Pseudospark Discharges with a Multi-Fluid Model, Proc. of the IX Int. Conf. on Gas Discharges and their Applications, Venice, Italy, 673 (1988)

5. K. Mittag, W.Niessen, Numerical Simulation of a Pseudospark Gas Discharge, Kerntechnik 52, 188 (1988)

6. A. J. Davies, C. J. Evans, P. M. Woodison, Simulation of the Growth of Axially Symmetric Discharges Between Plane Parallel Electrodes, Computer Physics Communications 14, 287 (1978)

7. A. J. Davies, Discharge Simulation, IEEE Proceedings, Vol. 133. Pt. A, No. 4, 217 (1986)

8. E. Halter, Die Berechnung elektrostatischer Felder in Pulsłeitungsanlagen, Kernforschungszentrum Karlsruhe, KfK-Report 4072 (1986)

9. W. Niessen, Monte-Carlo-Simulation of the Prebreakdown Phase for a high E/N-Discharge, Proc. of the IX Int. Conf. on Gas Discharges and their Applications, Venice, Italy, 670 (1988)

10. Y. Kaufman, P.Choi, private communication (1989)

11. P. Choi, H. Chuaqui, J. Lunney, R. Reichle, A. J. Davies, K. Mittag, Plasma Formation in a Pseudospark Discharge, IEEE Transaction for Plasma Science, to be published

12. D. Dietrich, C. Schultheiss, K. Mittag, to be published 


\section{Acknowledgement}

Stimulating discussions with W. Bauer, P.Choi, A. J. Davies, W. Hartmann, Y. Kaufman, W. Niessen, and C. Schultheiss were valuable and encouraging for this work, which was initiated by $W$. Schmidt. Especially the constructive criticism by $P$. Choi is appreciated. The parallel plate version of the code to solve the continuity equations was supplied together with considerable introductary help by $A$. J. Davies. The Poisson solver was contributed by E. Halter, who also was very helpful in incorporating it into the other code. Y. Kaufman updated the coefficients for ionization and photon emission after a thorough literature search. The plots were produced using computer routines by $\mathrm{K}$. Thurnay who was never tired of answering my questions. 


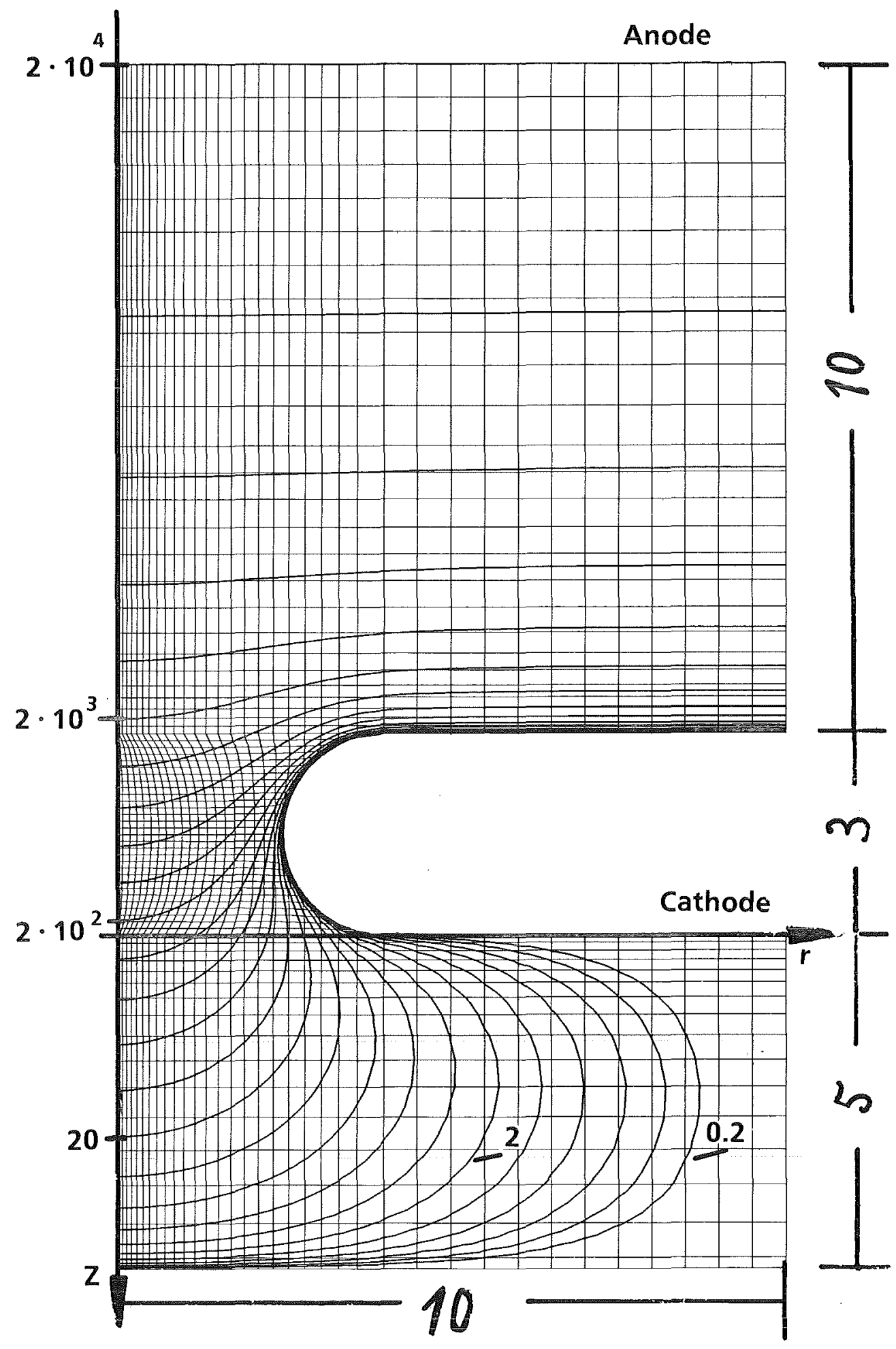

Fig. 1. Pseudospark geometry (mm), numerical mesh, and equipotential lines (Volt) 


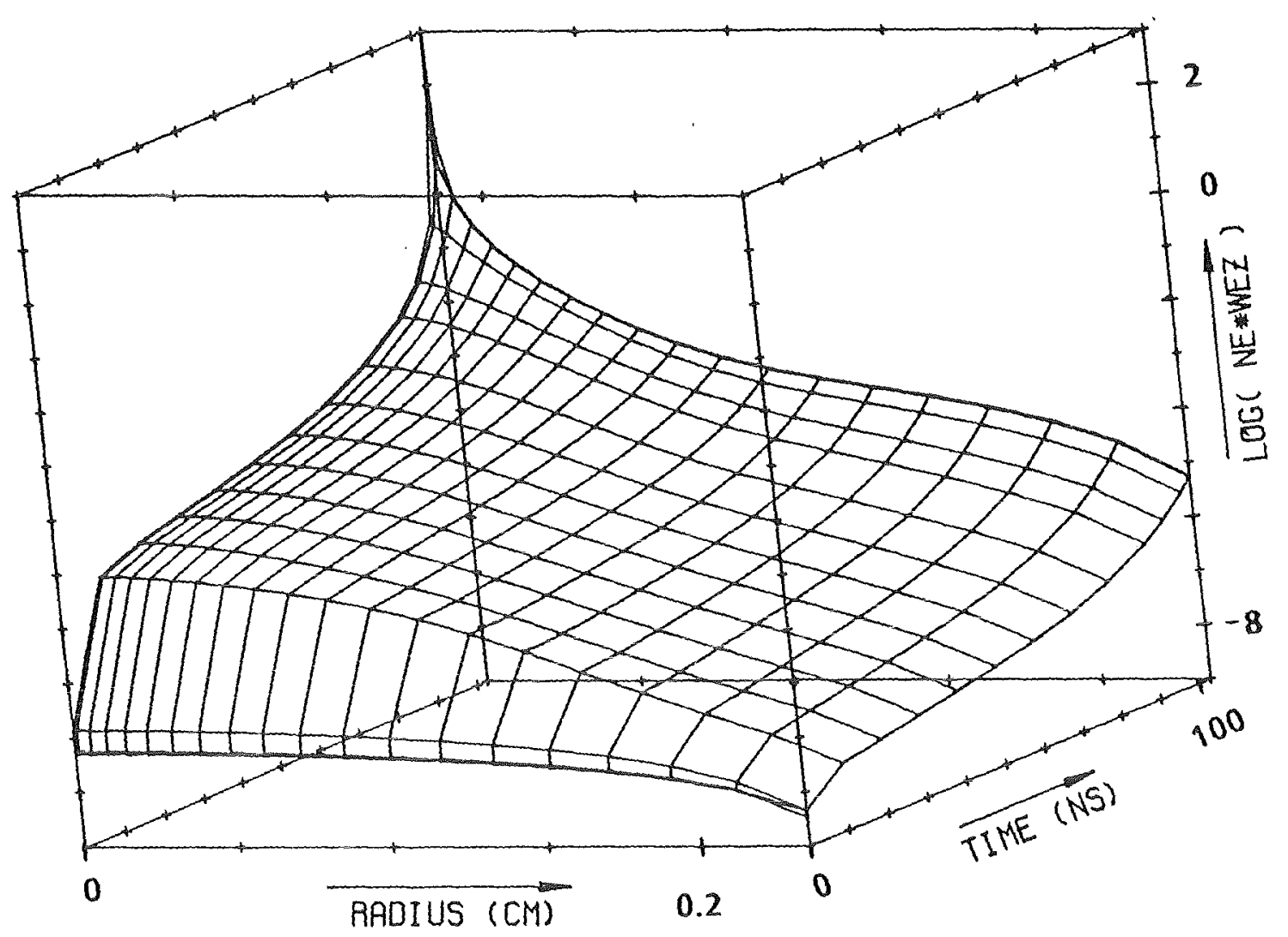

FIG. 2 . CURRENT DENSITY AT THE HOLE FOR BOUNDARY MODEL (1) IN A/CM 2

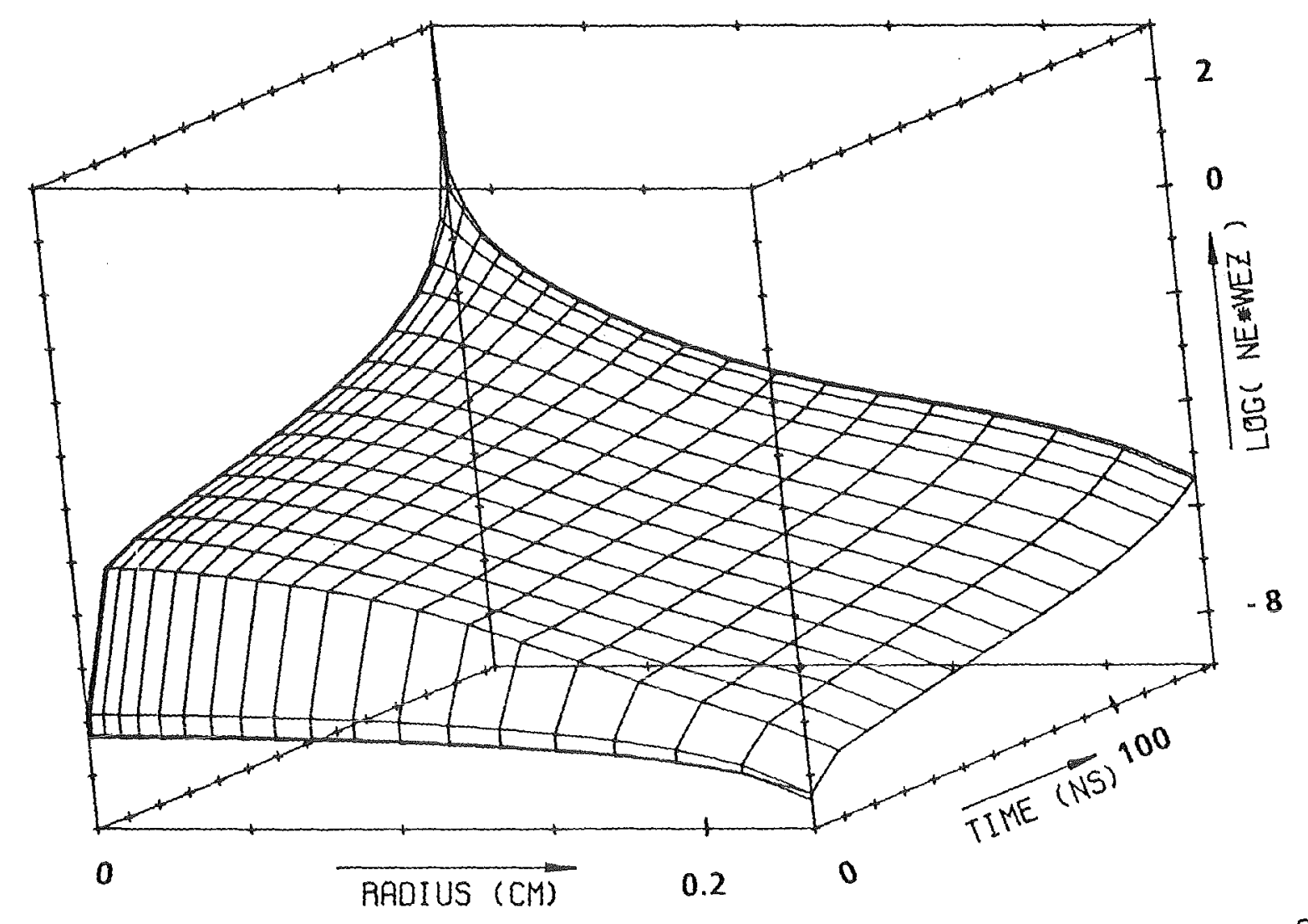

FIG. 3 . CURRENT DENSITY AT THE HOLE FOR BOUNDARY MODEL (2) IN A/CMW: 


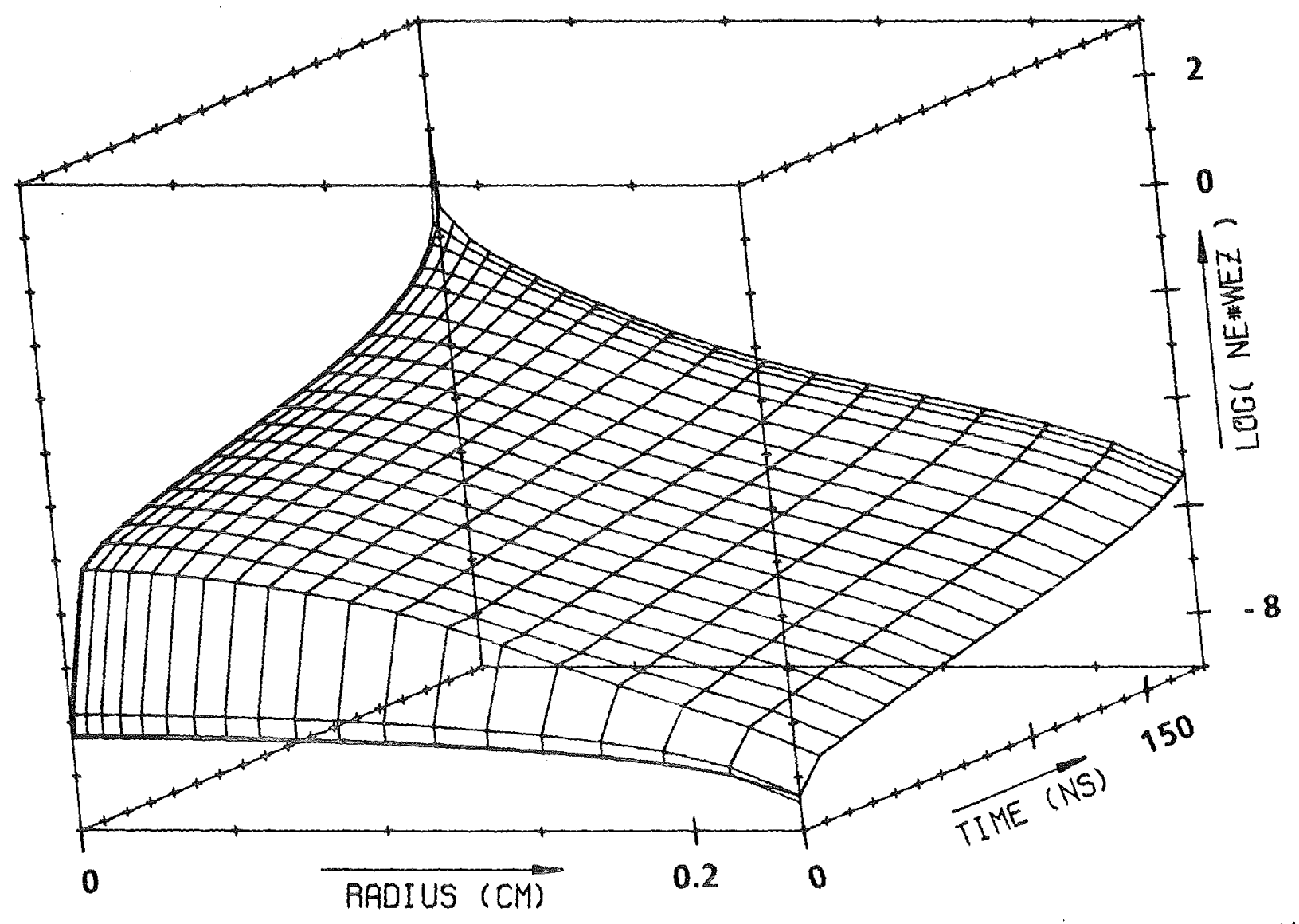




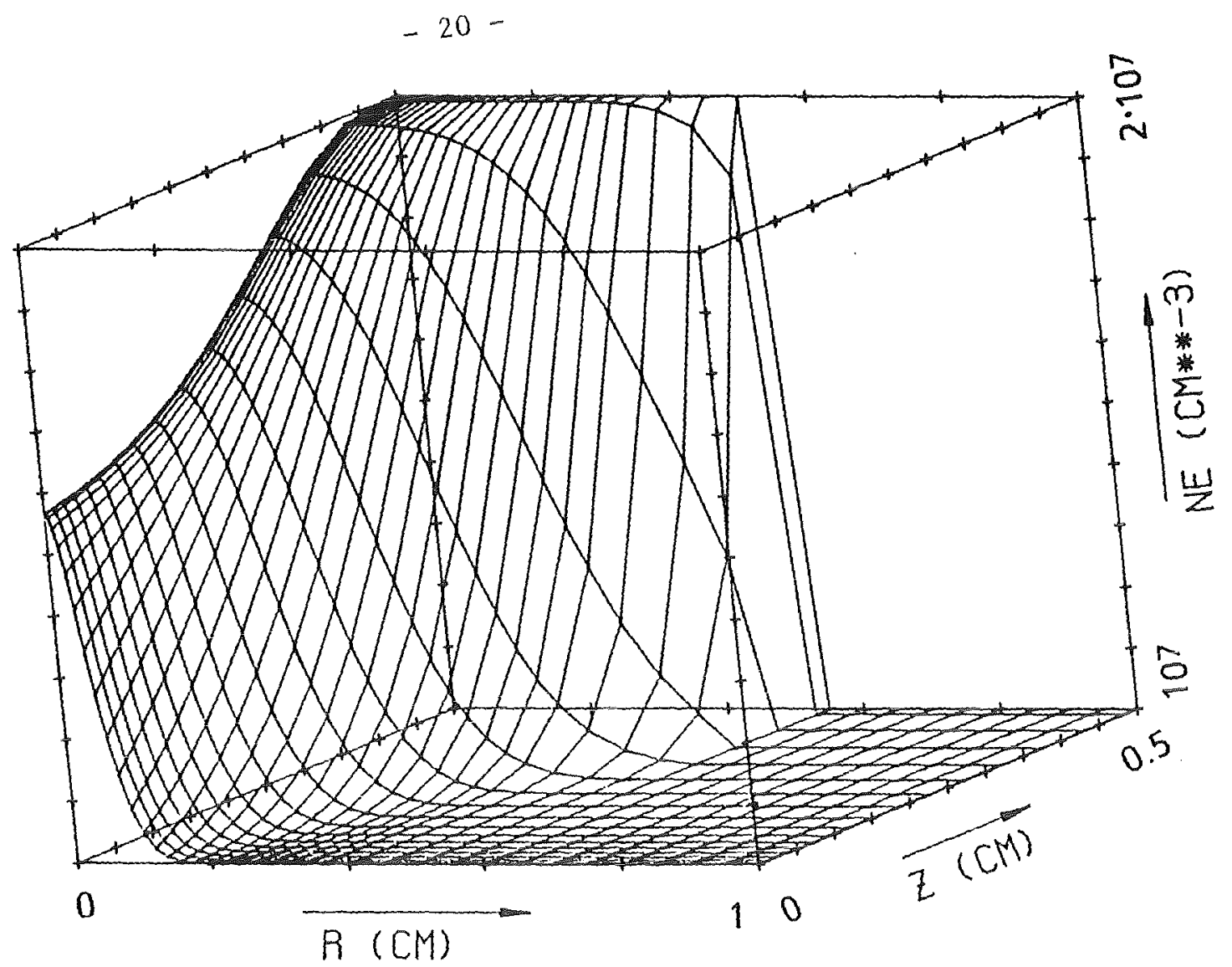

FIG. 5. ELECTRON DENSITY AFTER 1:0 ELECTRON PASSAGES ON AXIS

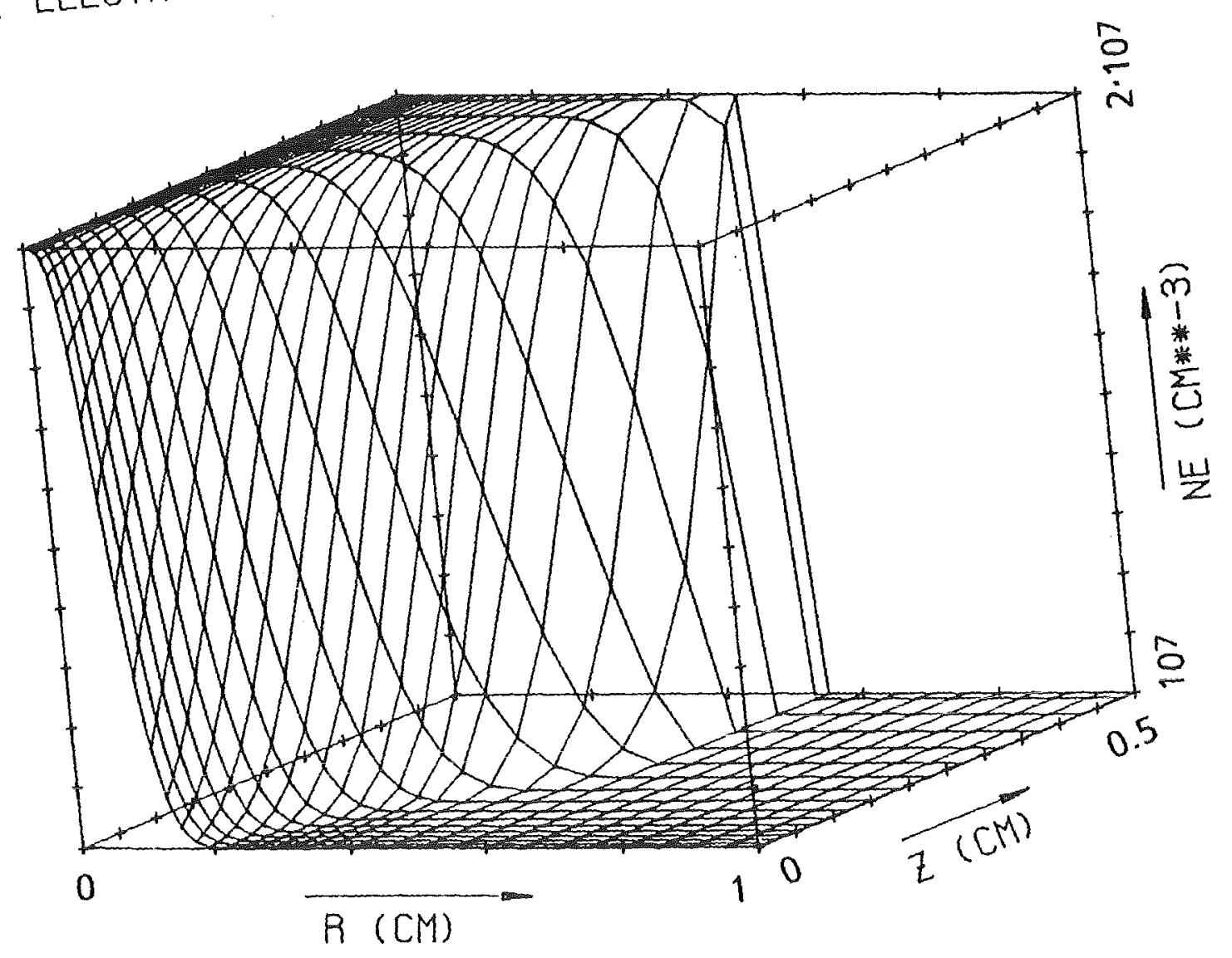

FIG. 6. ELECTRON DENSITY, TIME INDEPENDENT SOLUTION 


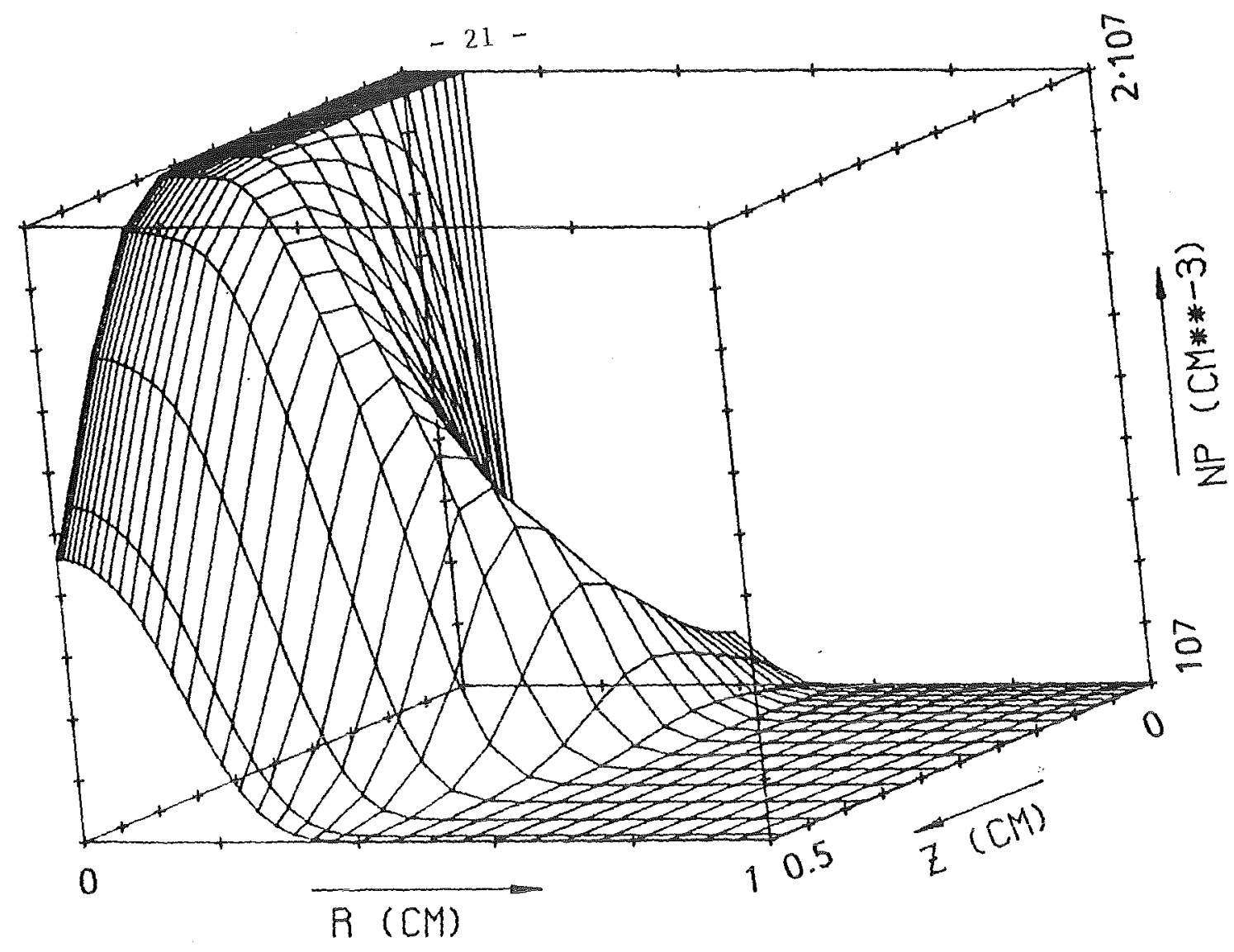

FIG. 7 . ION DENSITY AFTER 1.0 ION PASSAGES ON AXIS

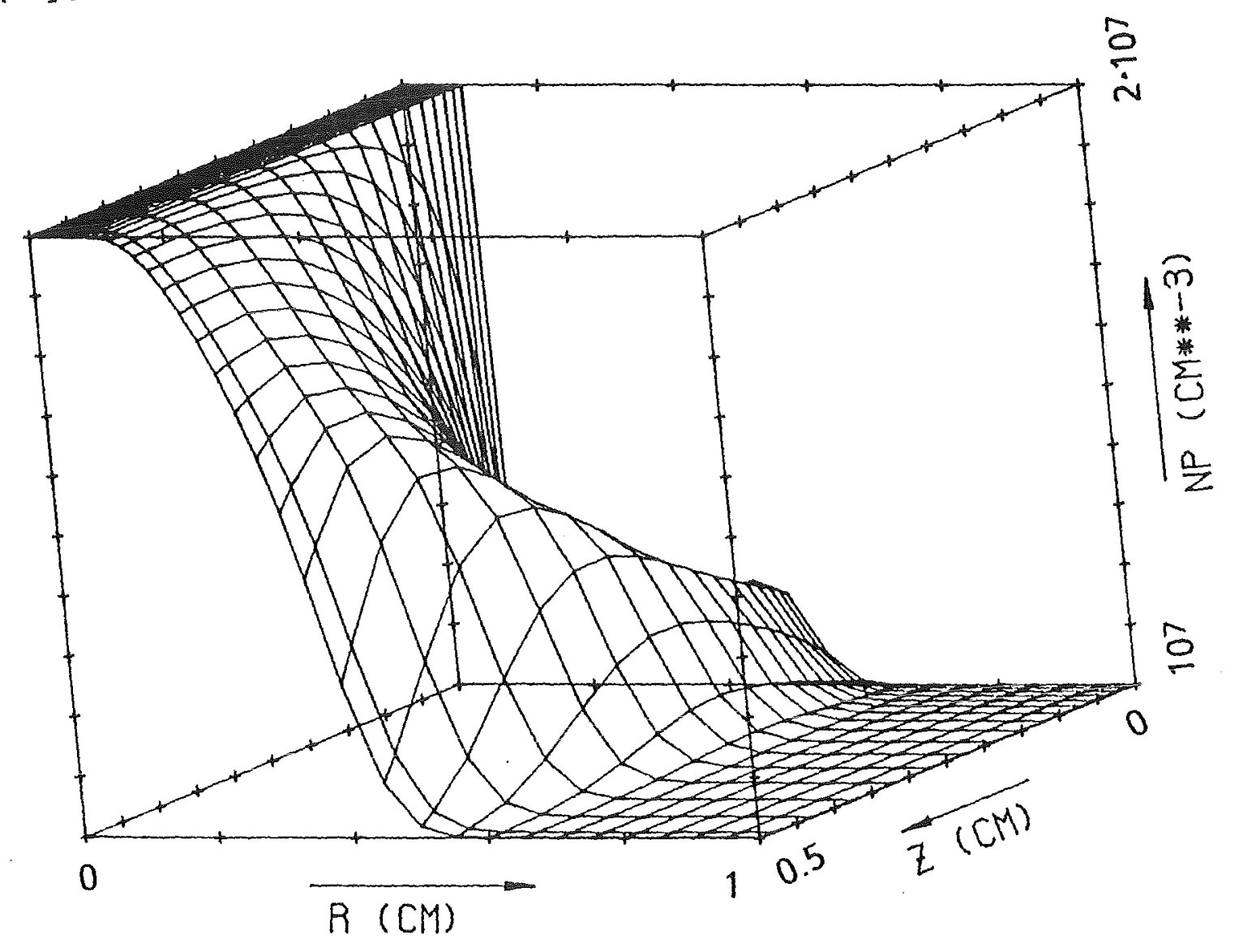

FIG. 8. ION DENSITY AFTER 2.9 ION PASSAGES ON AXIS 


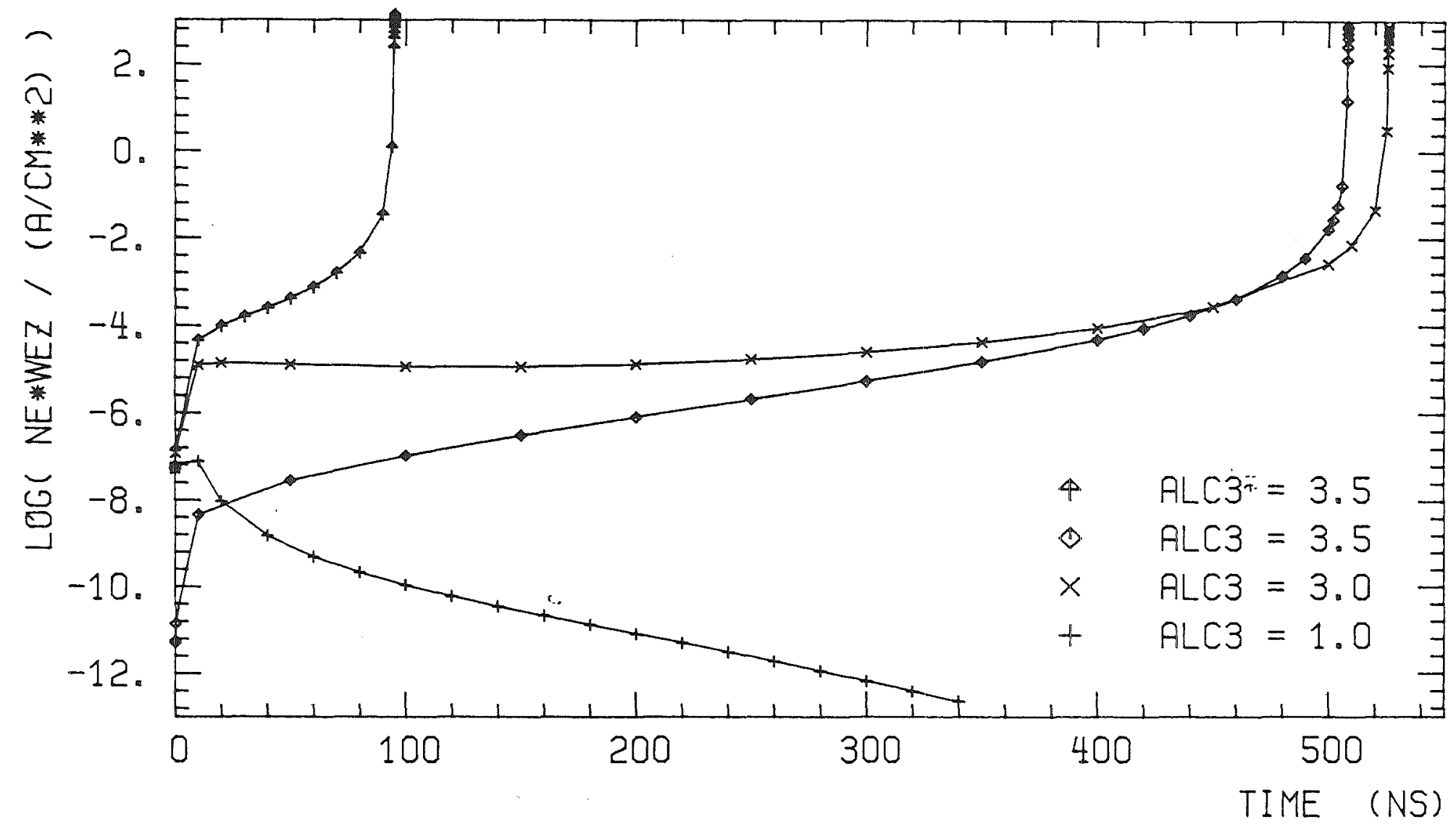

FIG. 9 . CURRENT DENSITY ON AXIS ON HOLLOW CATHCDE BOUNDARY 


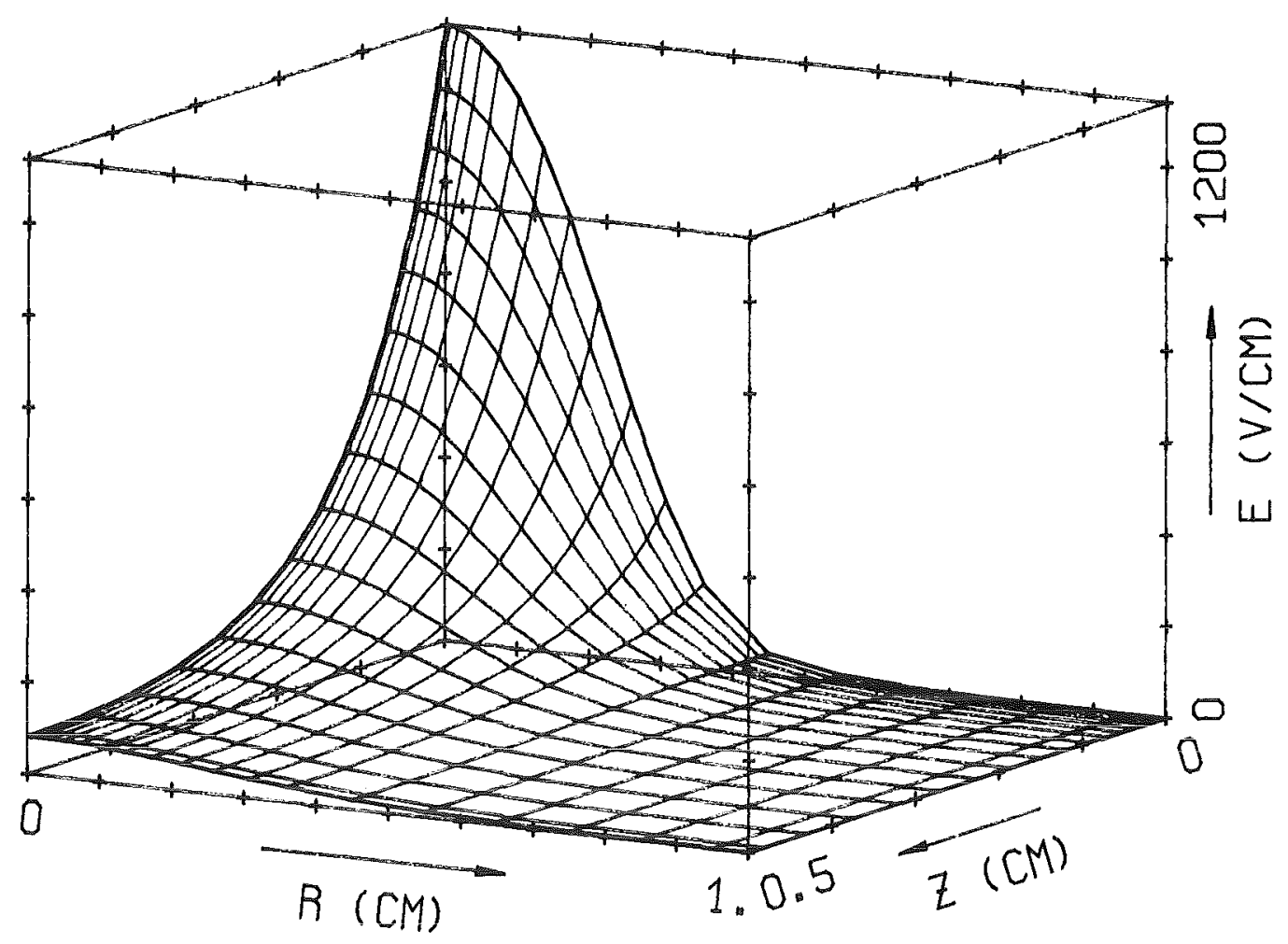

FIG. 10. ELECTRIC FIELD AT THE START OF THE SIMULATION T=0

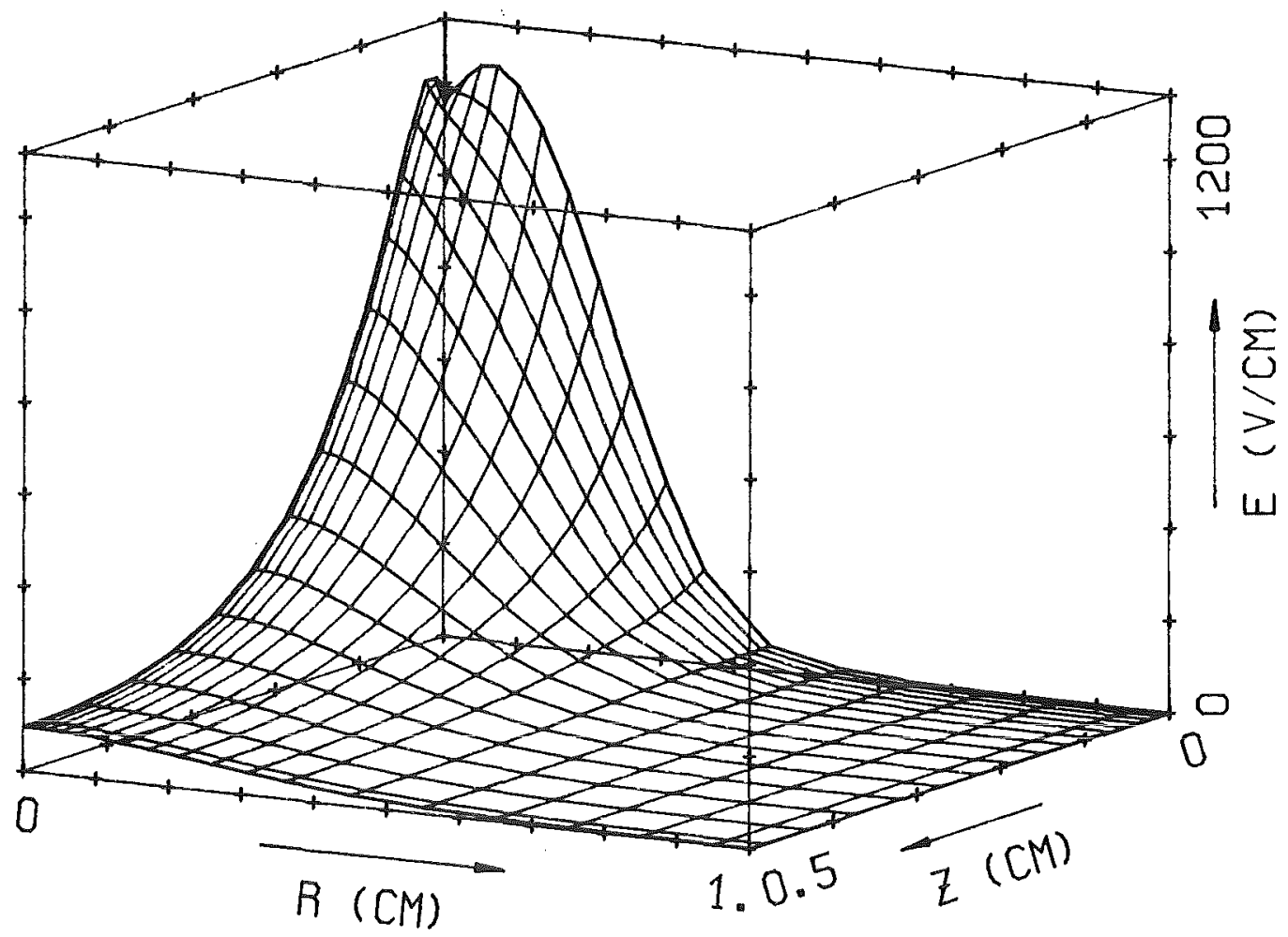

FIG. 13. ELECTRIC FIELD AT $T=508$ NS 


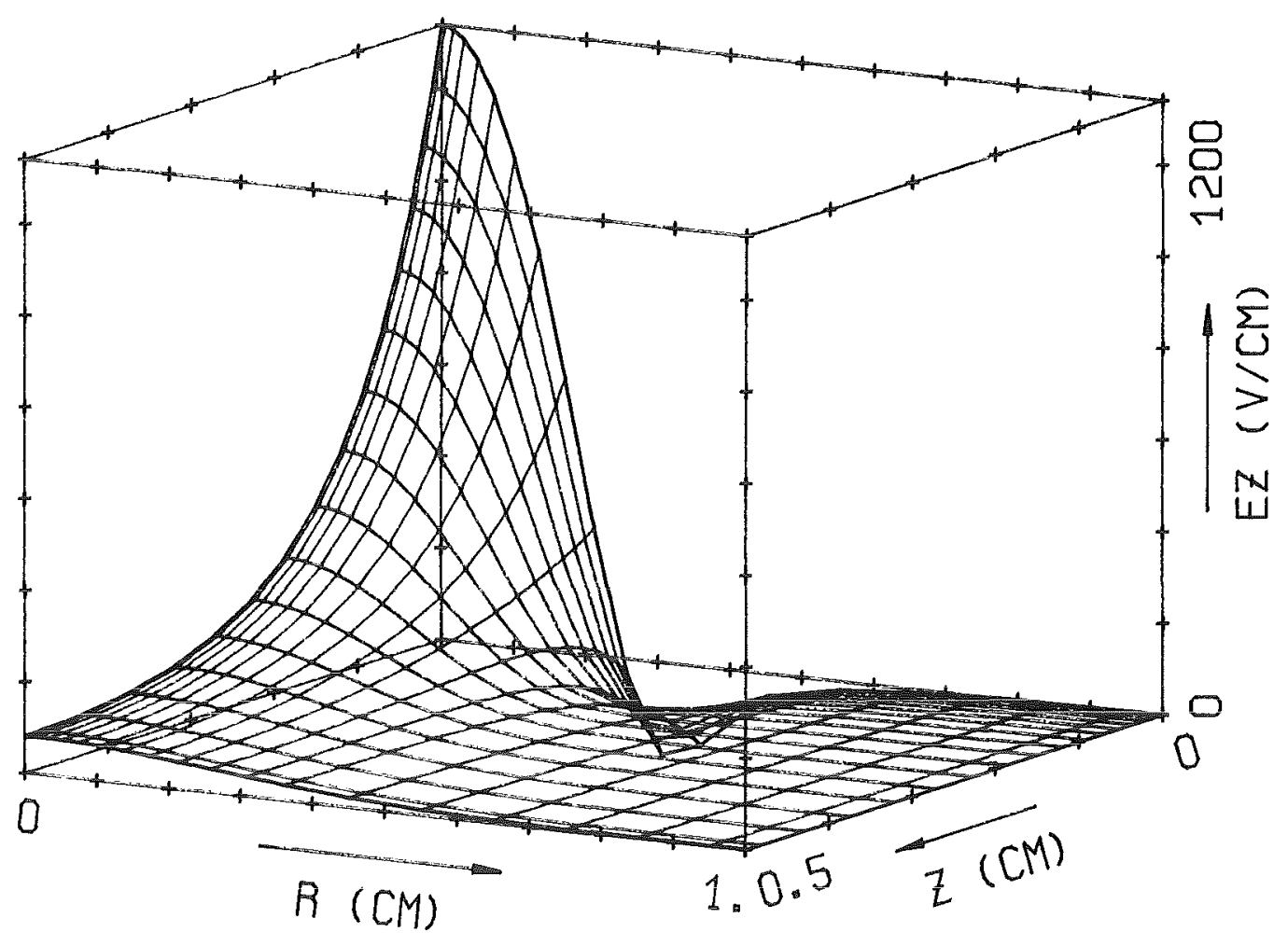

FIG. 11. AXIAL ELECTRIC FIELD AT $T=0$

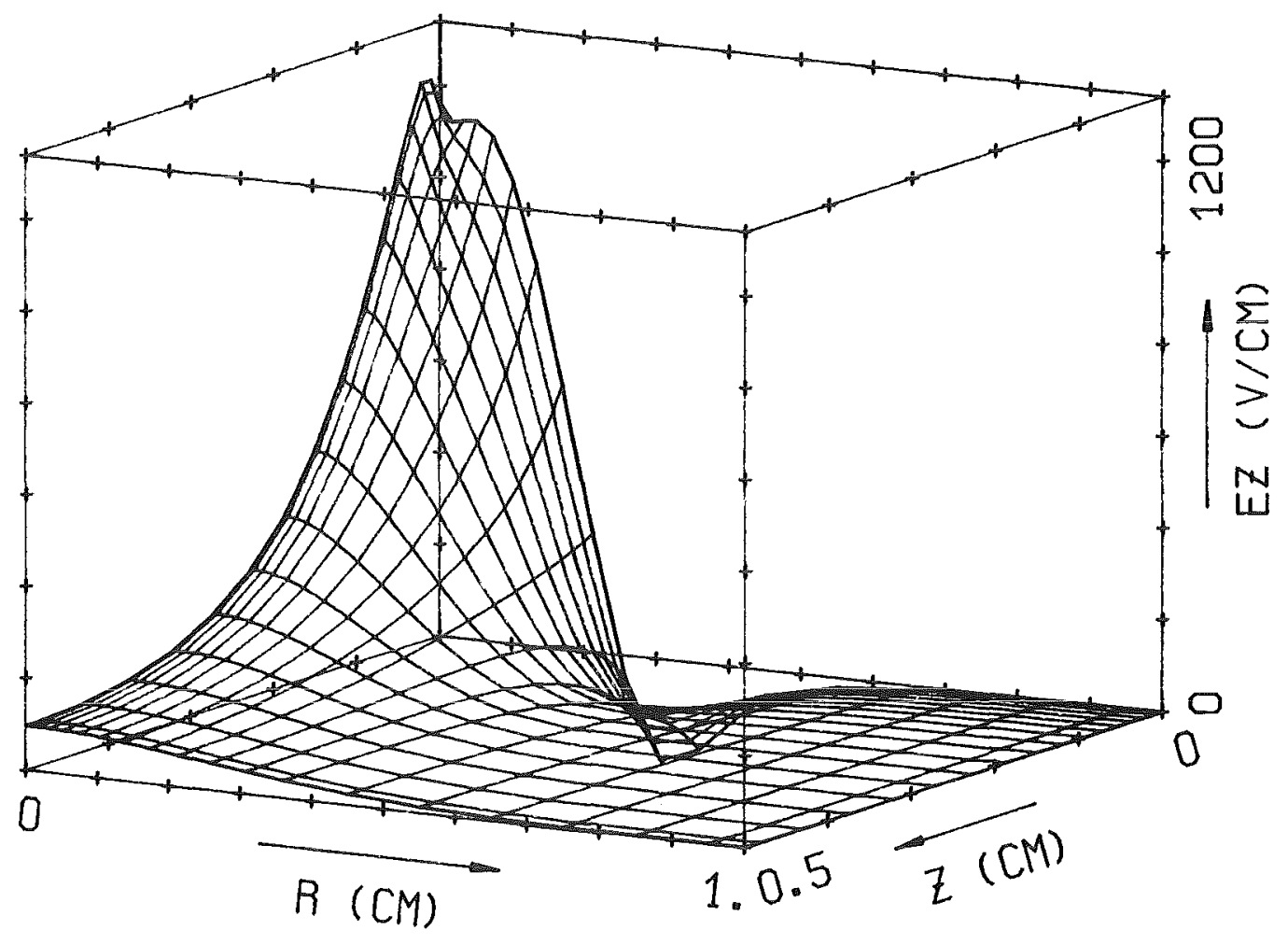

FIG. 14. AXIAL ELECTRIC FIELD AT $T=508$ NS 


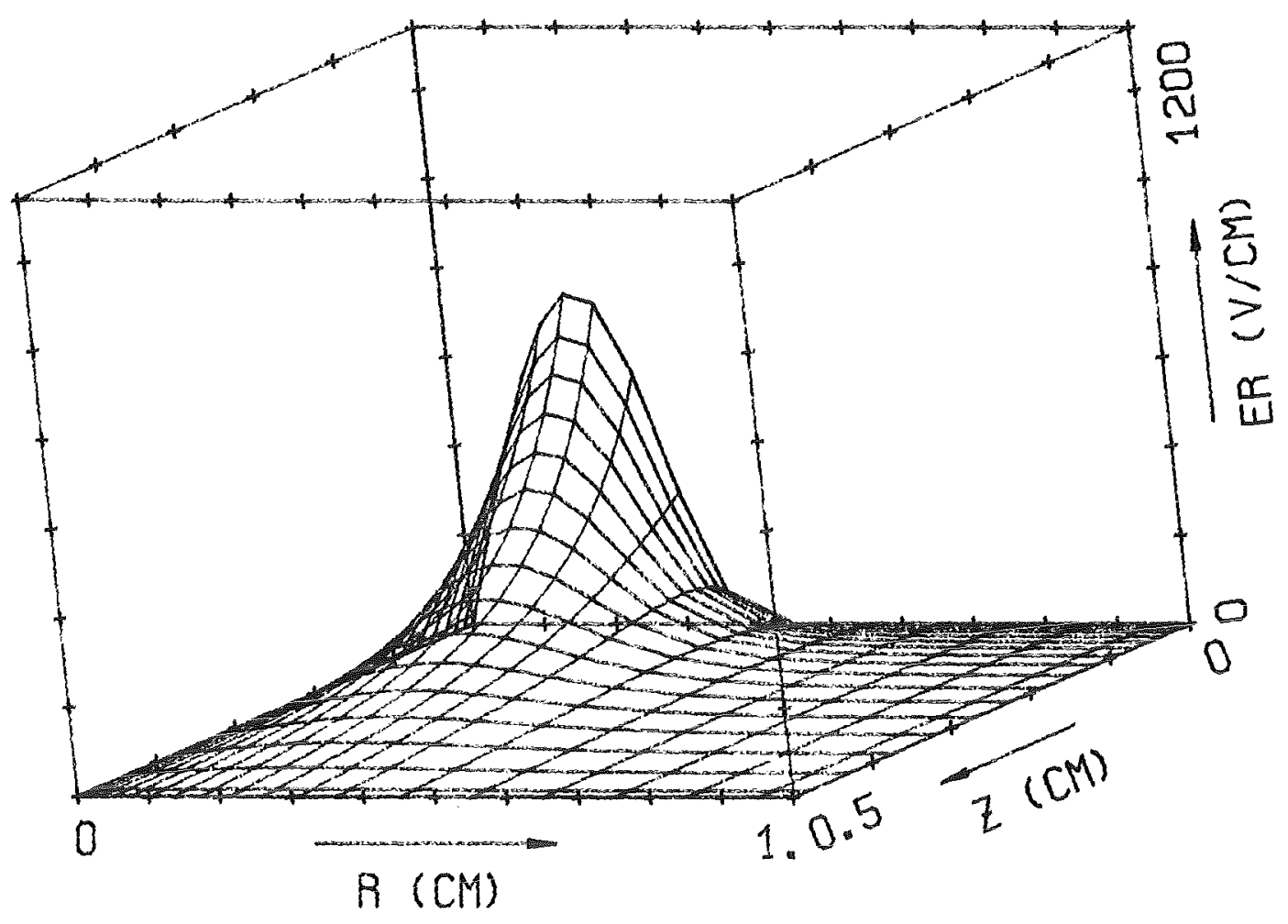

FIG. 12. RADIAL ELECTRIC FIELD AT $T=0$

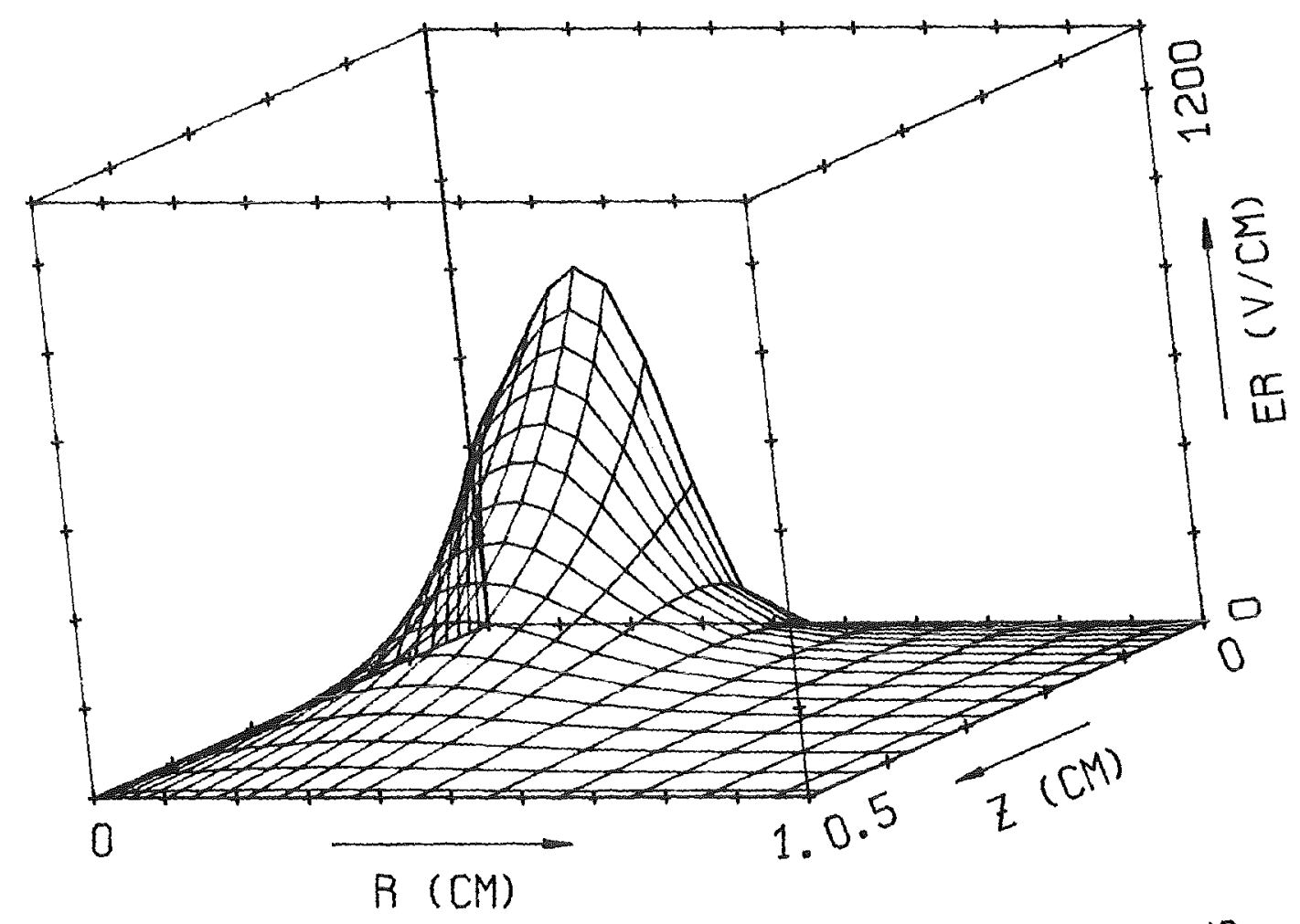

FIG. 15. RADIAL ELECTRIC FIELD AT $T=508 \mathrm{NS}$ 


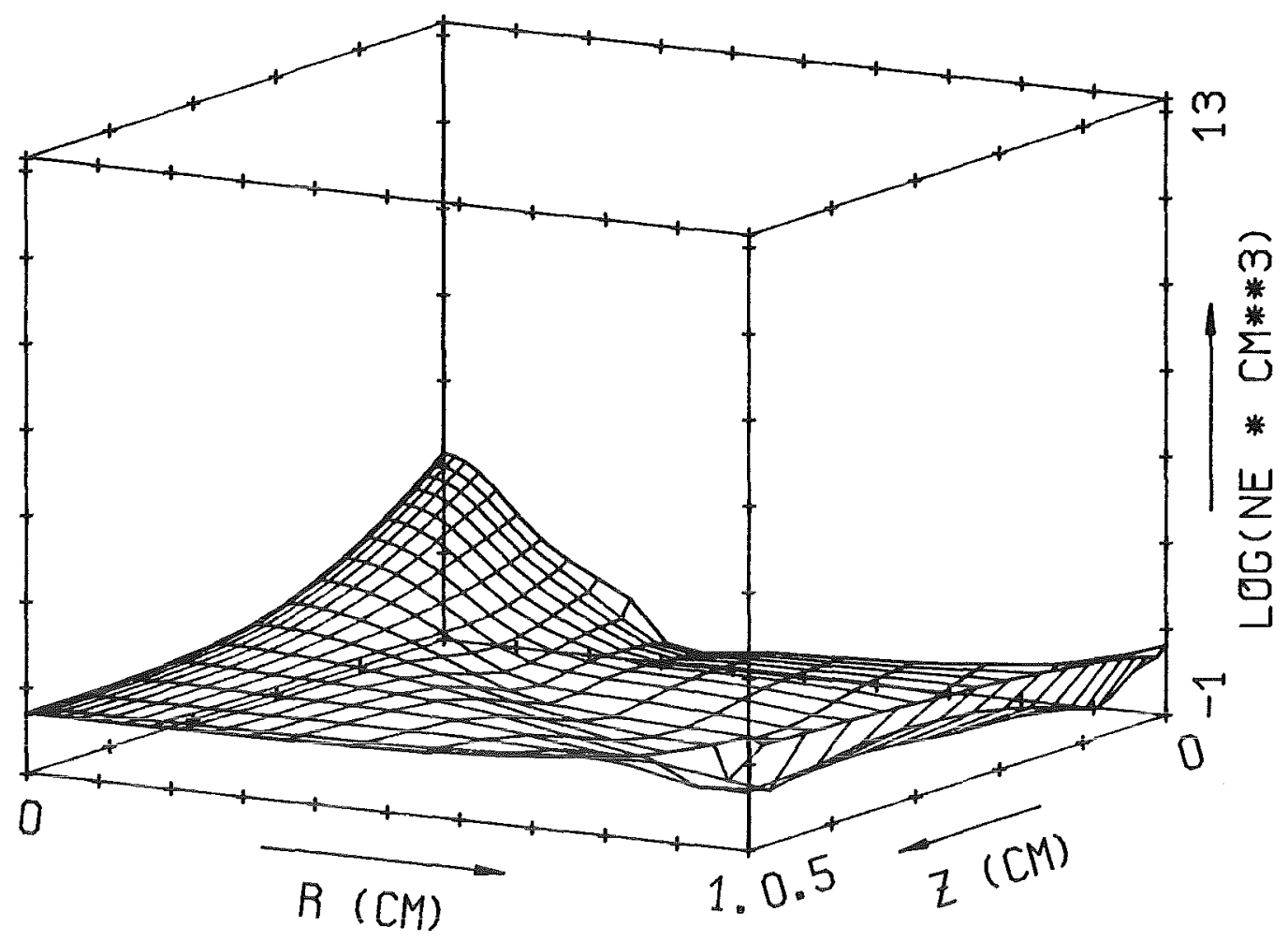

FIG. 16 . ELECTRON DENSITY AT $T=100$ NS

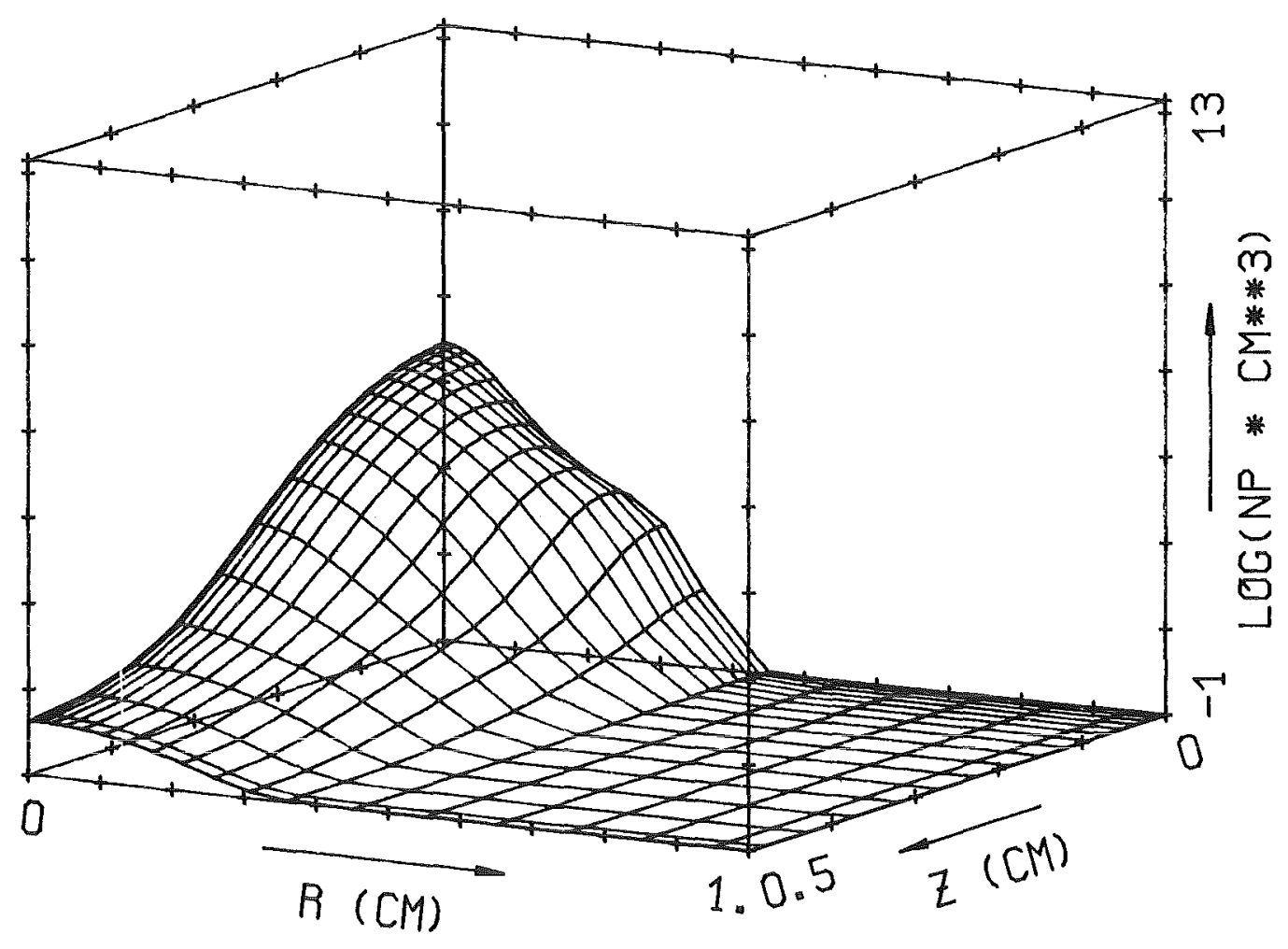

FIG. 17. POSITIVE ION DENSITY AT $T=100 \mathrm{NS}$ 
$-27-$

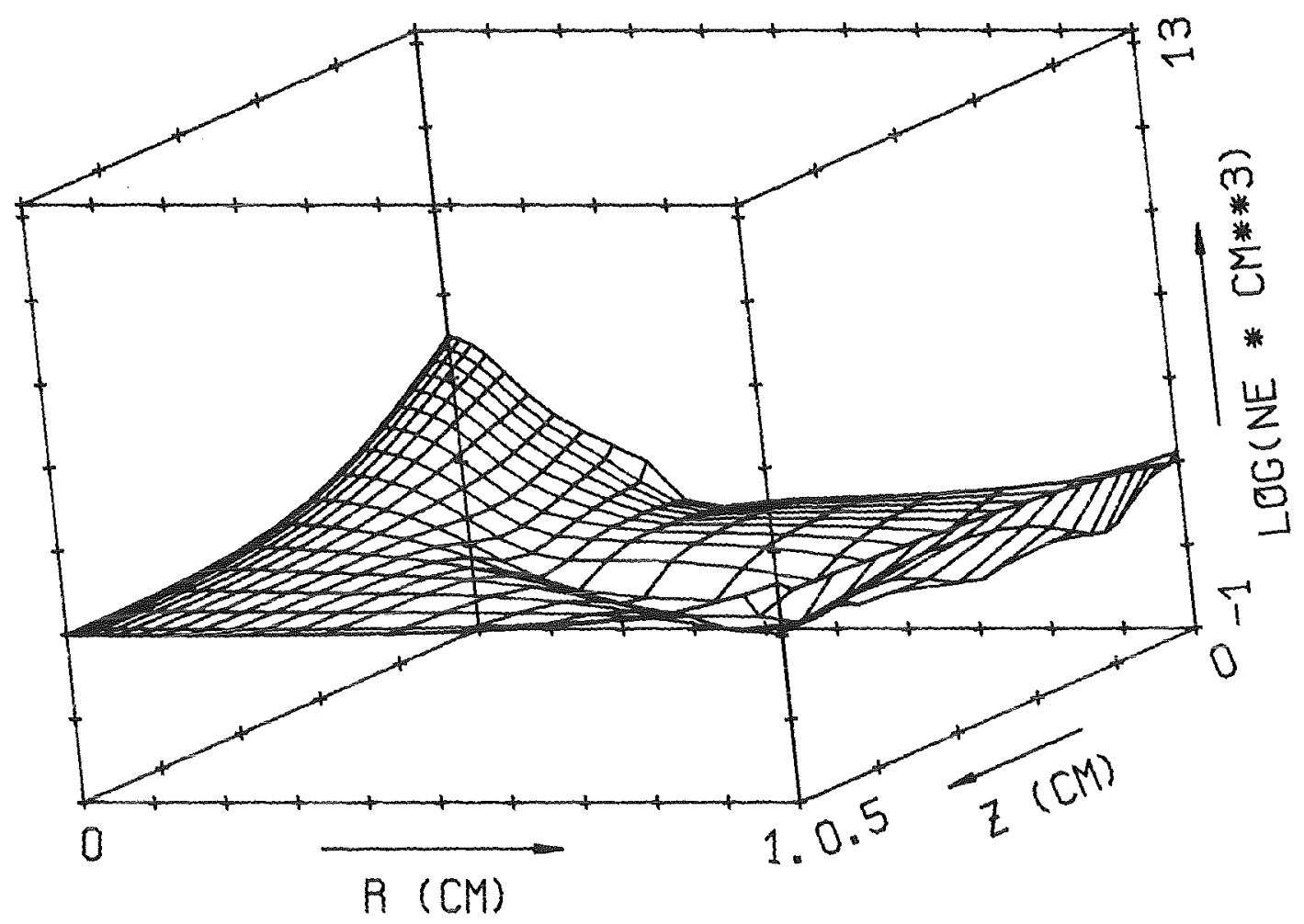

FIG. 18. ELECTRON DENSITY AT $T=400 \mathrm{NS}$

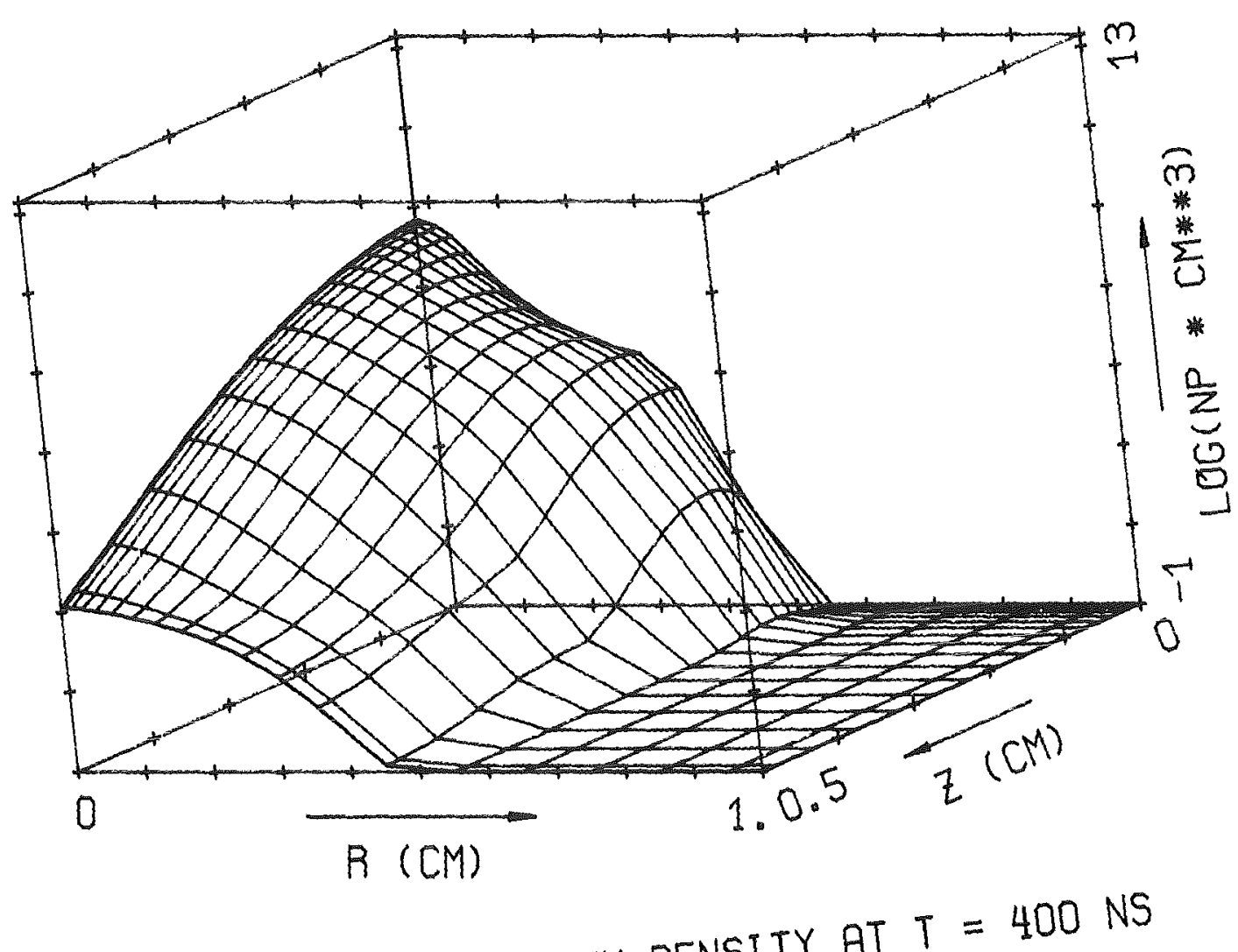

FIG. 19. POSITIVE ION DENSITY AT $T=400$ NS 


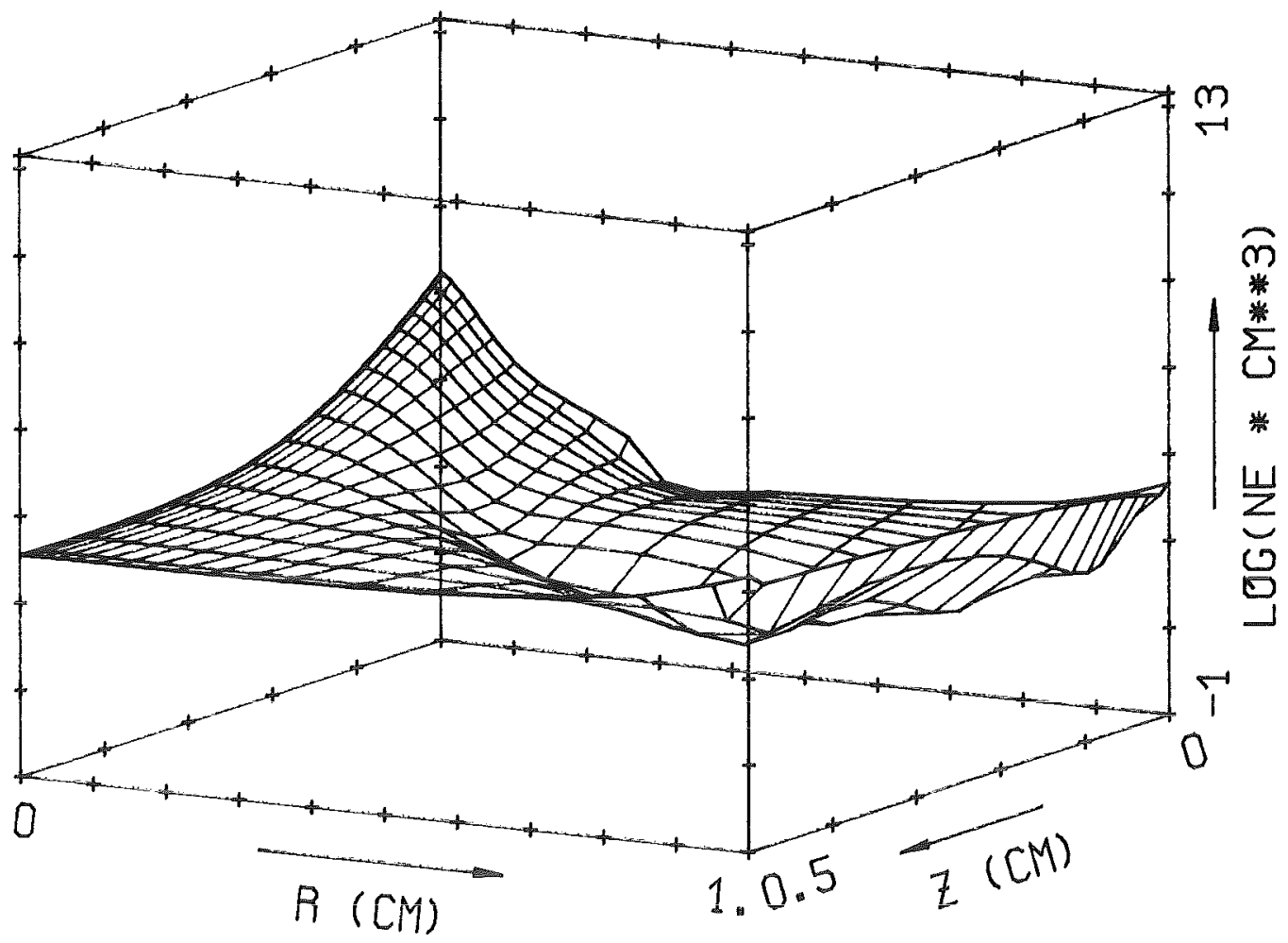

FIG. 20. ELECTRON DENSITY AT $T=480 \mathrm{NS}$

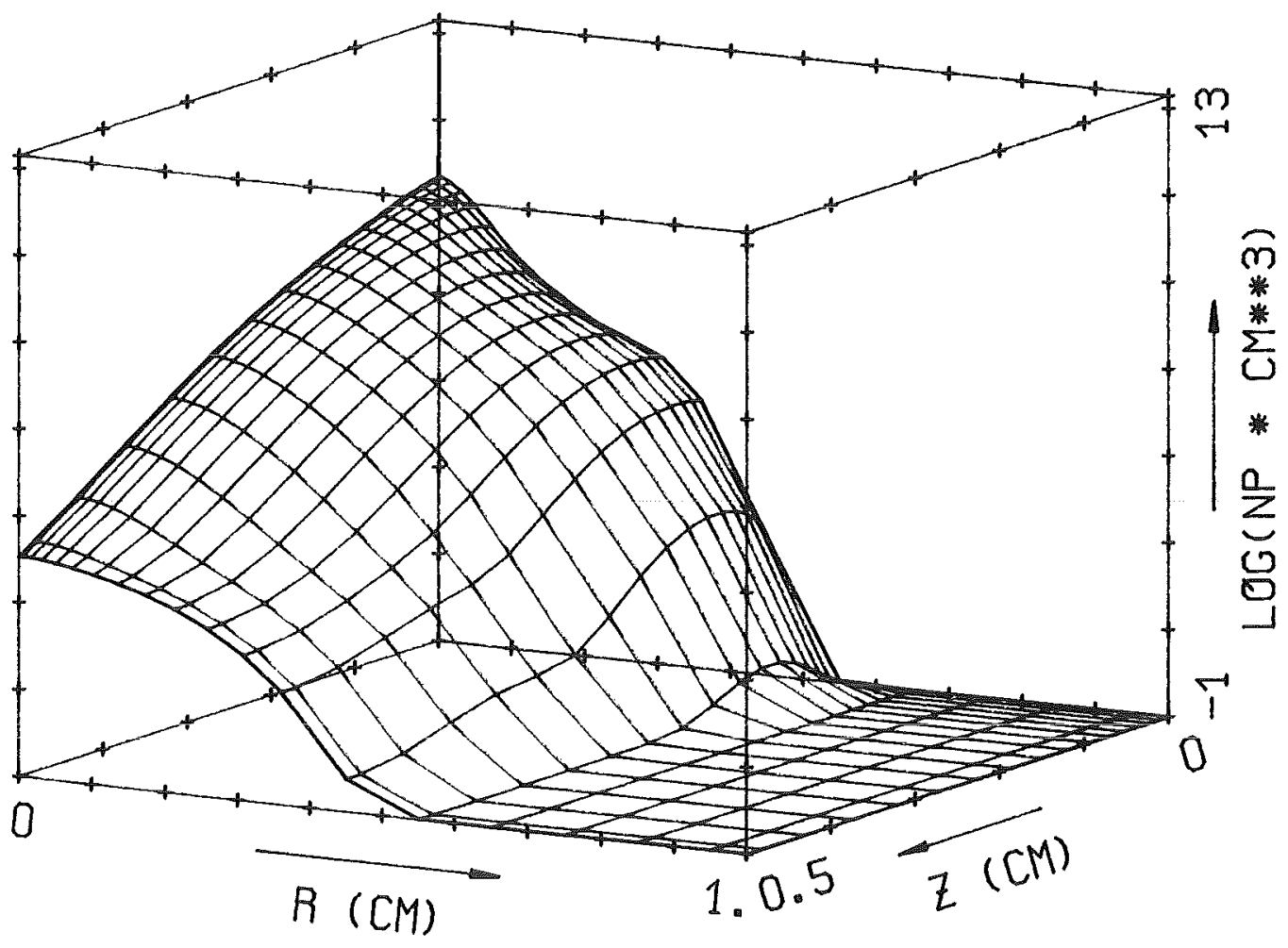

FIG. 21. POSITIVE ION DENSITY AT $T=480$ NS 


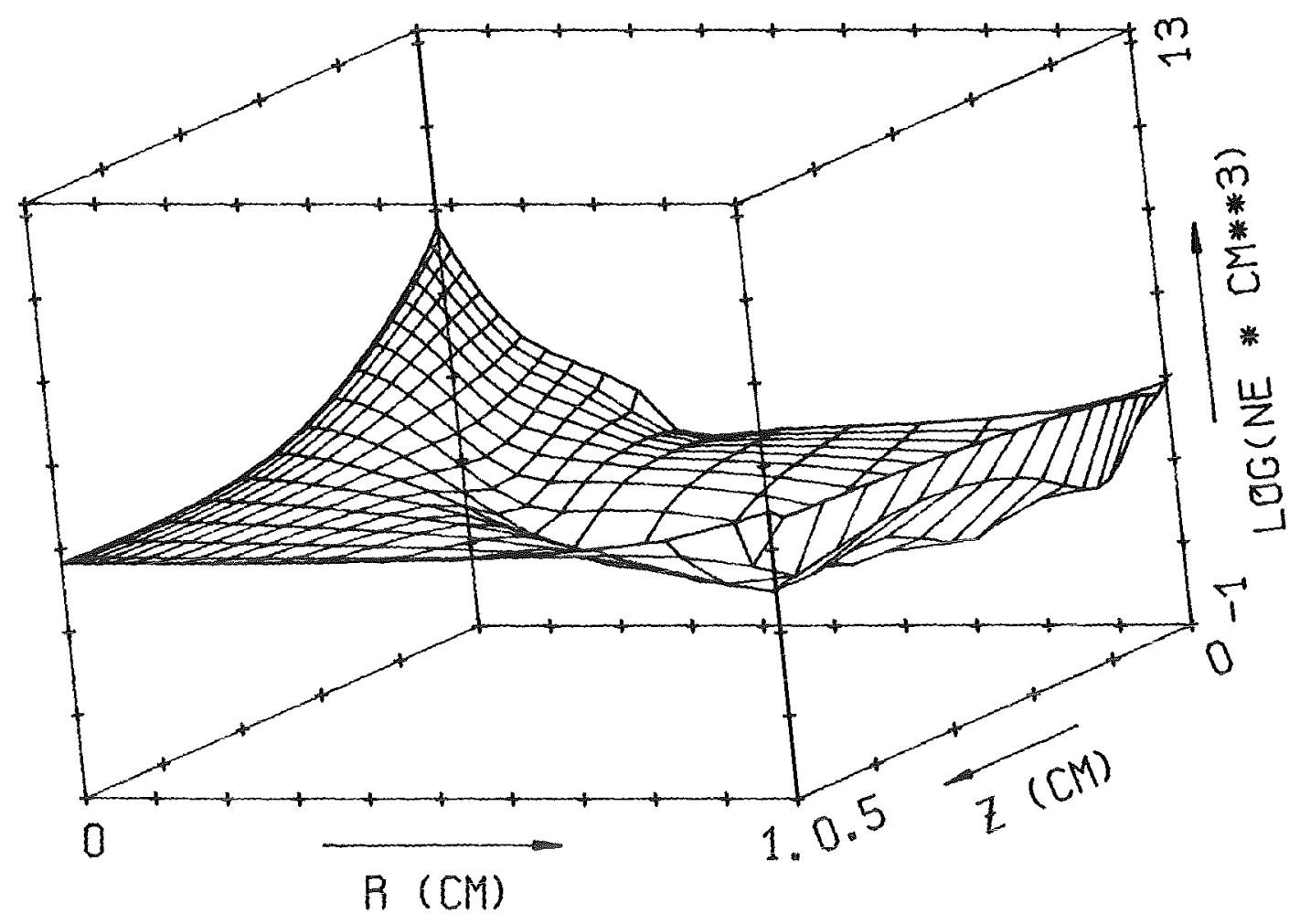

FIG. 22. ELECTRON DENSITY AT $T=500 \mathrm{NS}$

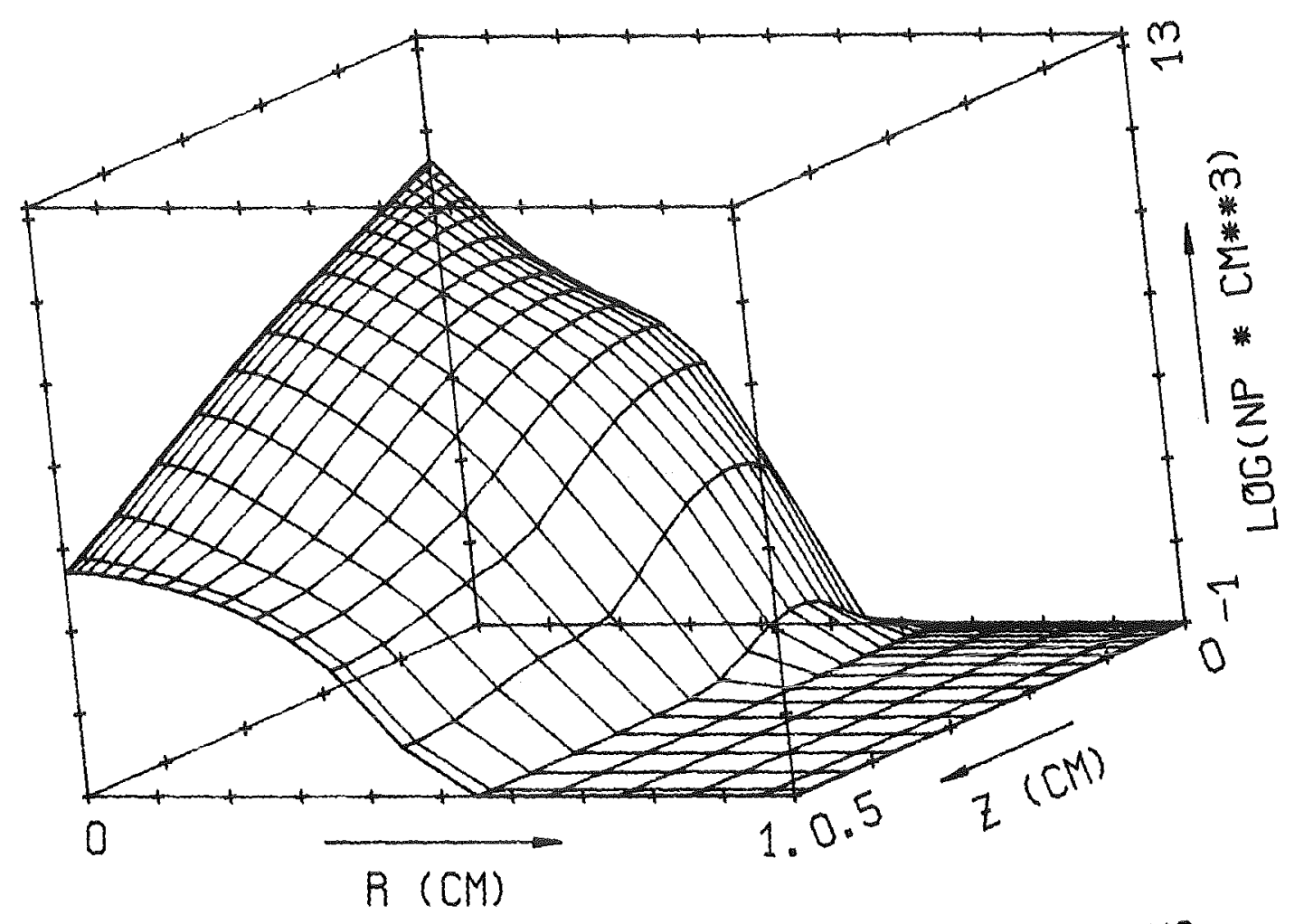

FIG. 23. POSITIVE ION DENSITY AT $T=500 \mathrm{NS}$ 
$-30-$

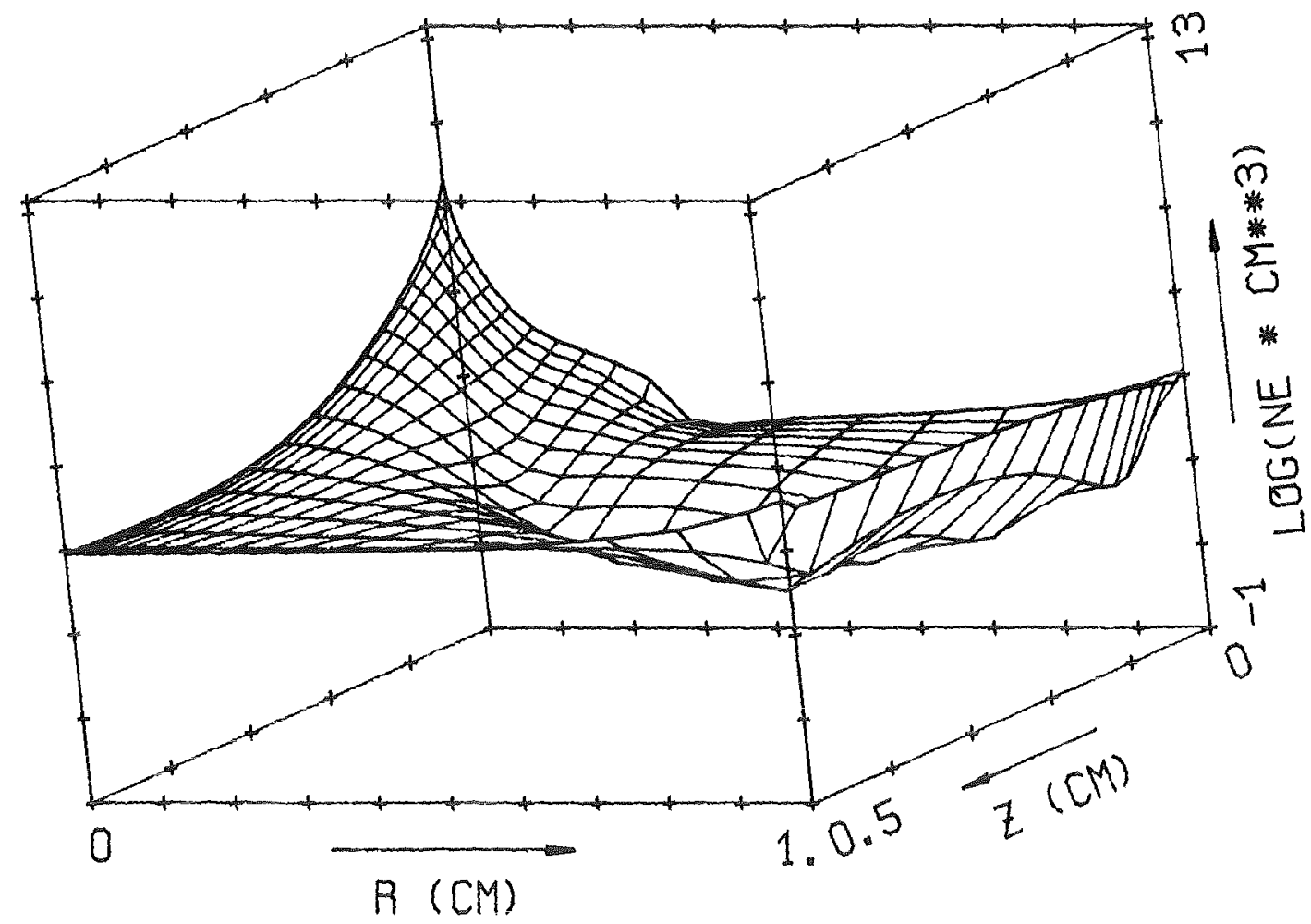

FIG. 24. ELECTRON DENSITY AT $T=506$ NS

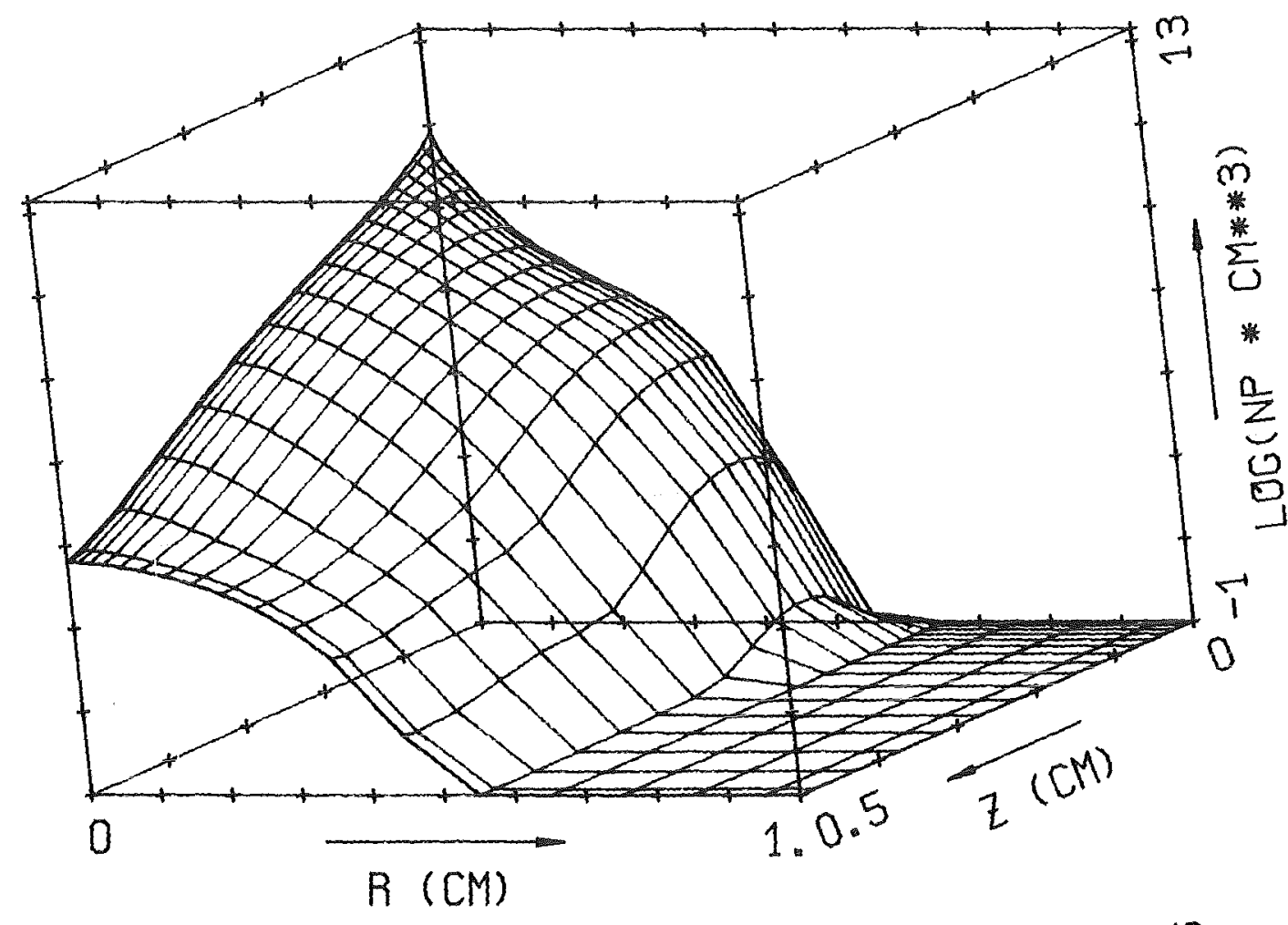

FIG. 25. POSITIVE ION DENSITY AT $T=506$ NS 


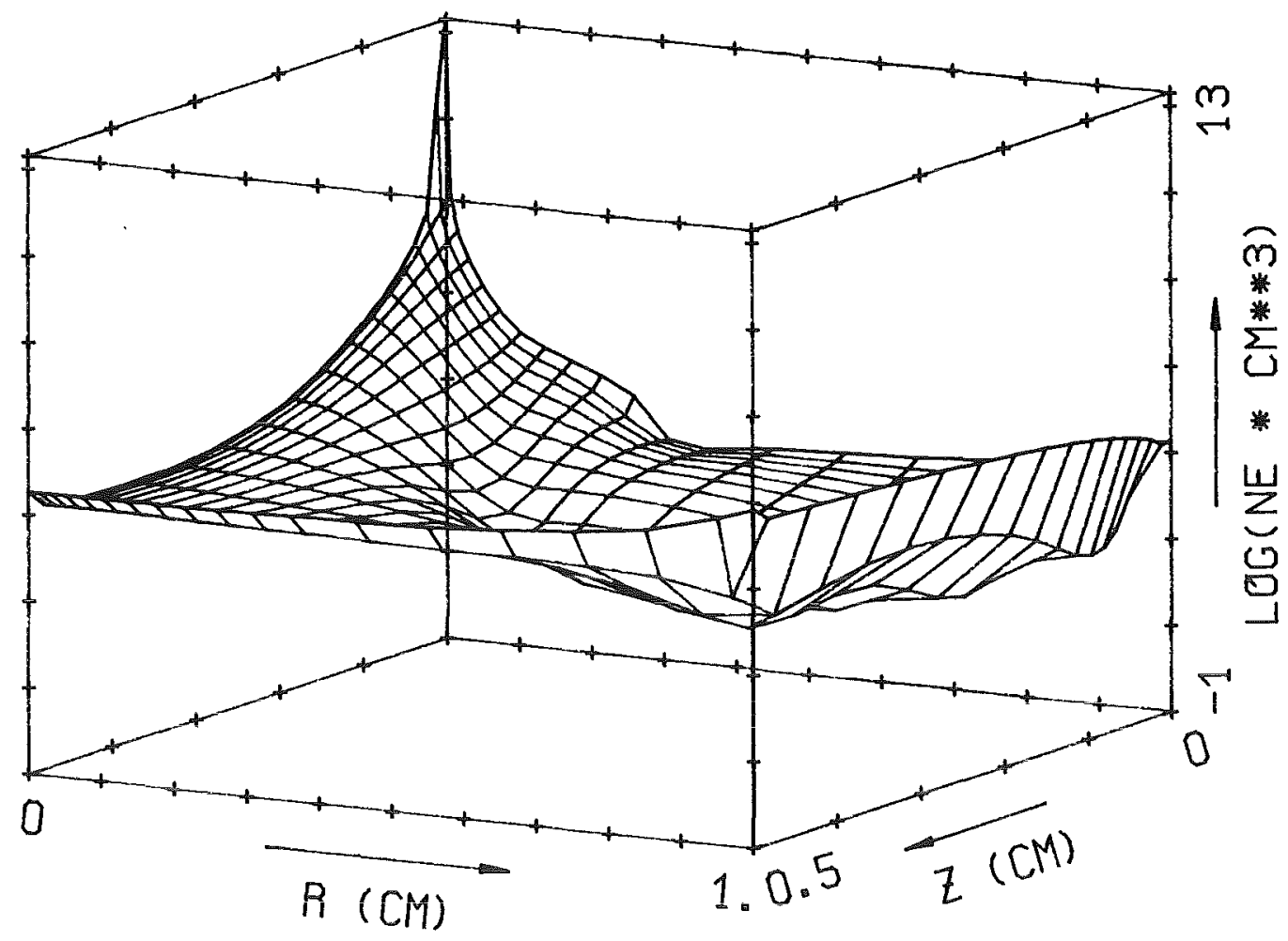

FIG. 26. ELECTRON DENSITY AT $T=508$ NS

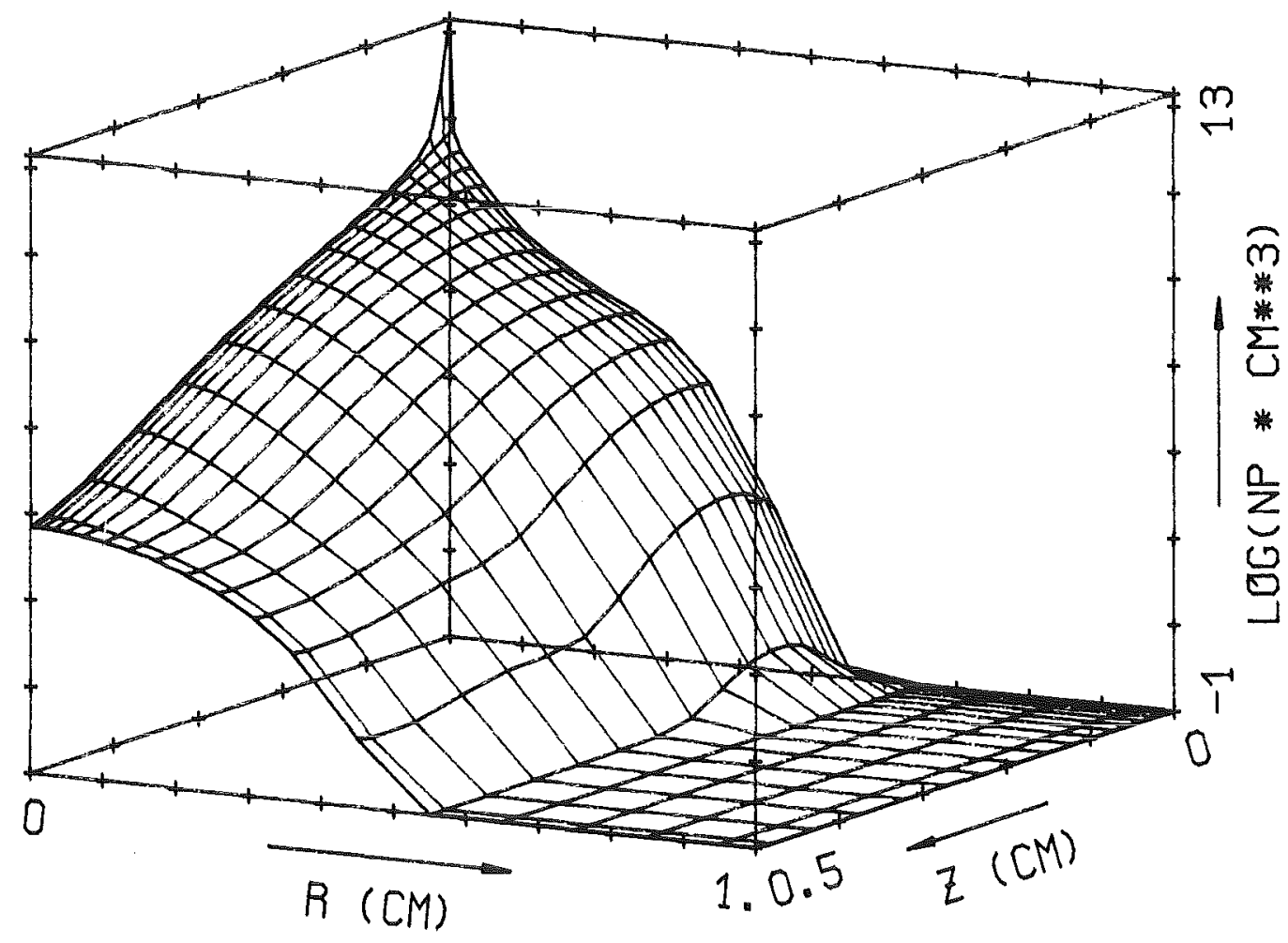

FIG. 27. POSITIVE ION DENSITY AT $T=508 \mathrm{NS}$ 


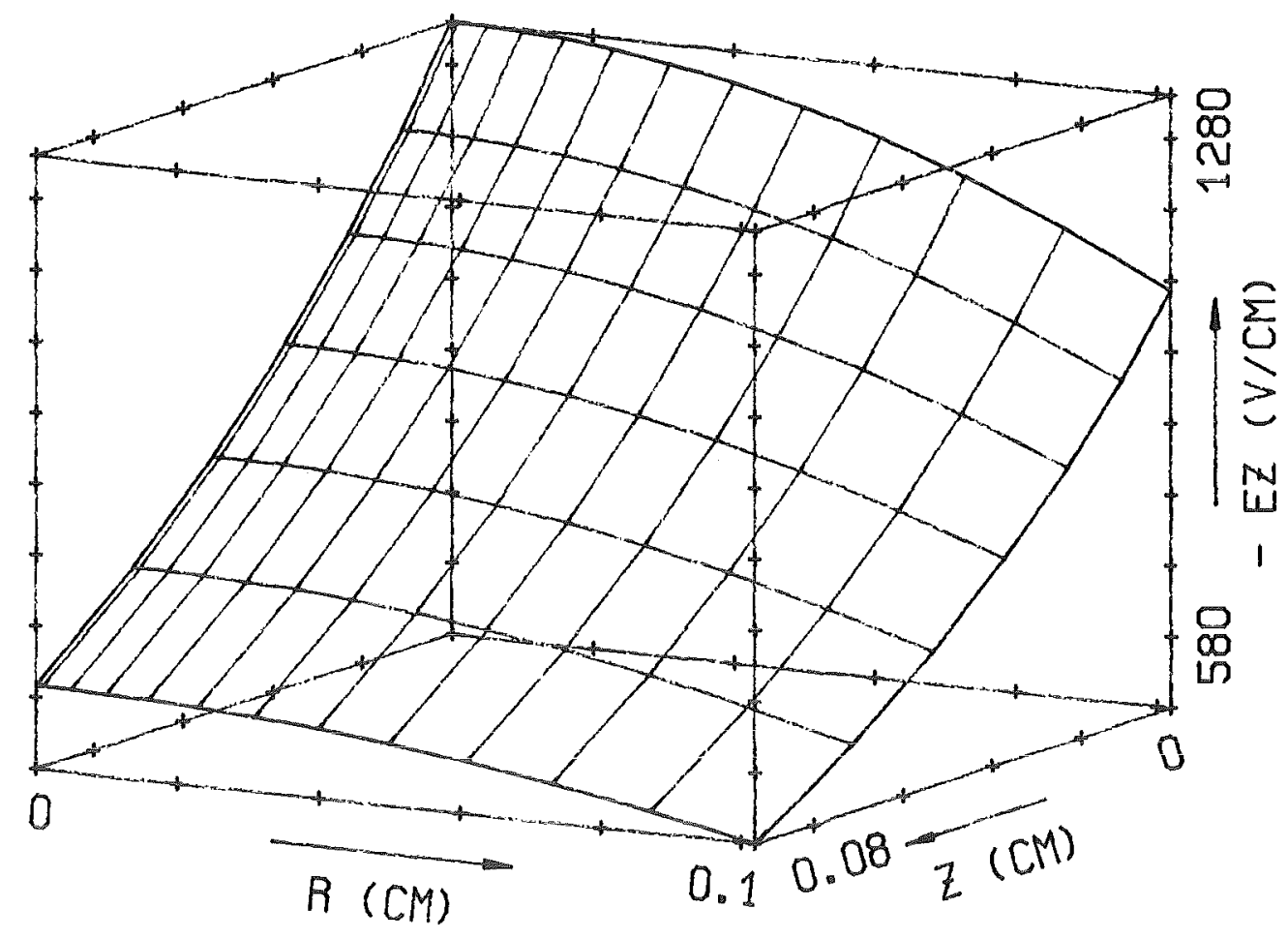

FIG. 28. - AXIAL ELECTRIC FIELD AT $T=0$

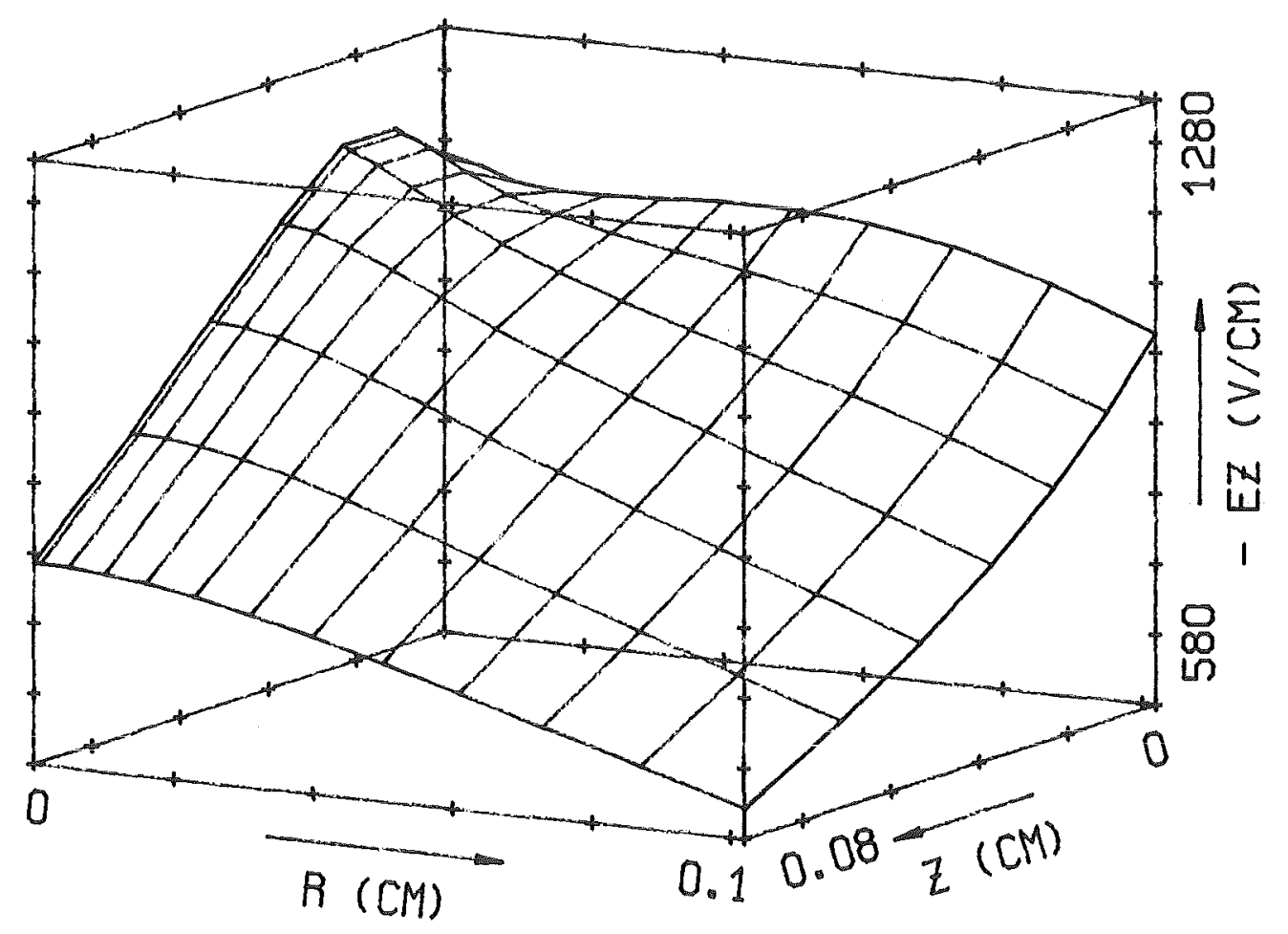

FIG. 30 - AXIAL ELECTRIC FIELD AT $T=508$ NS 


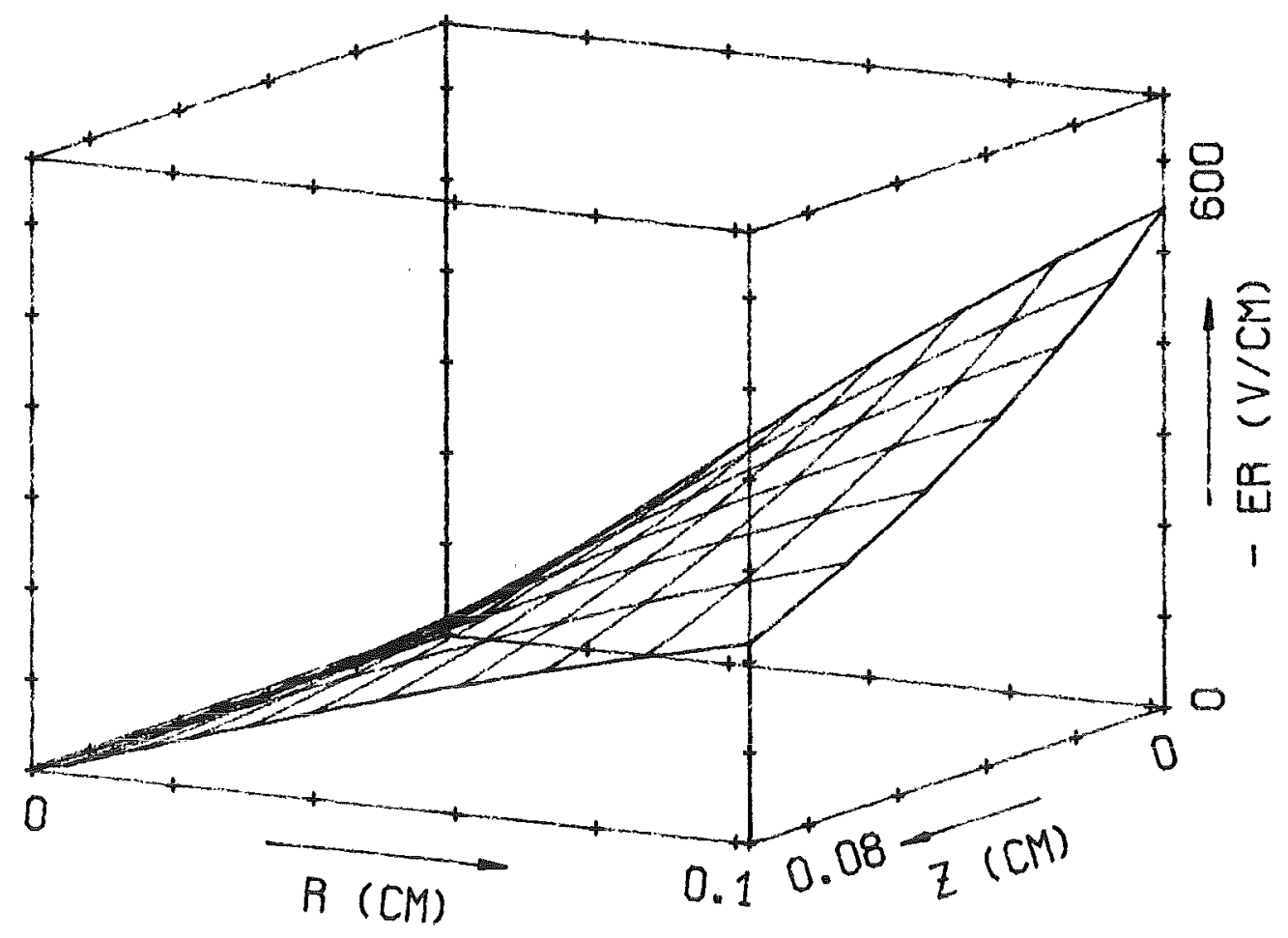

FIG. 29. - RADIAL ELECTRIC FIELD AT $T=0$

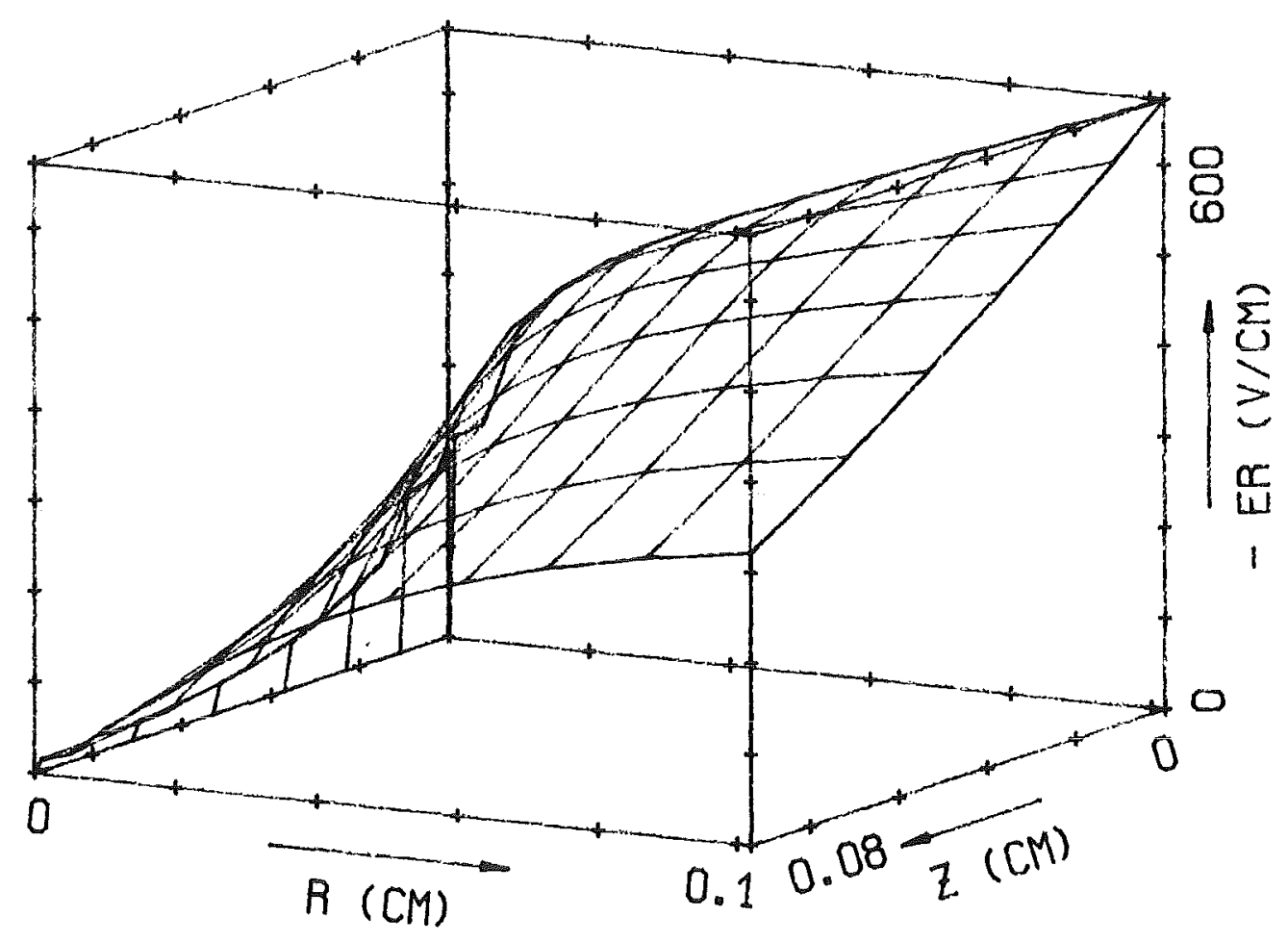

FIG. 31. - RADIAL ELECTRIC FIELD AT $T=508$ NS 


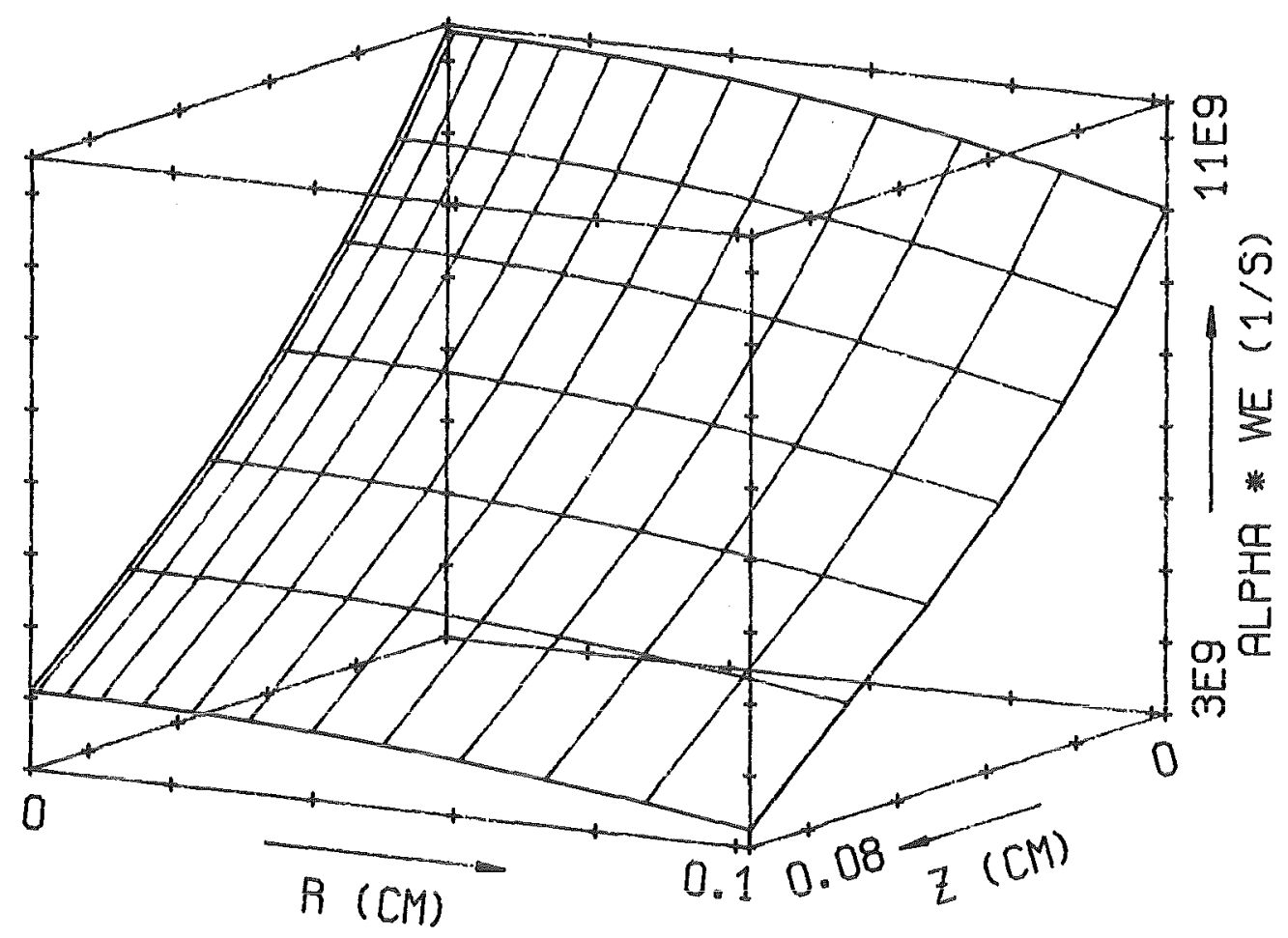

FIG. 32. IONIZATION COEFFICIENT * DRIFT VELOCITY AT T=0

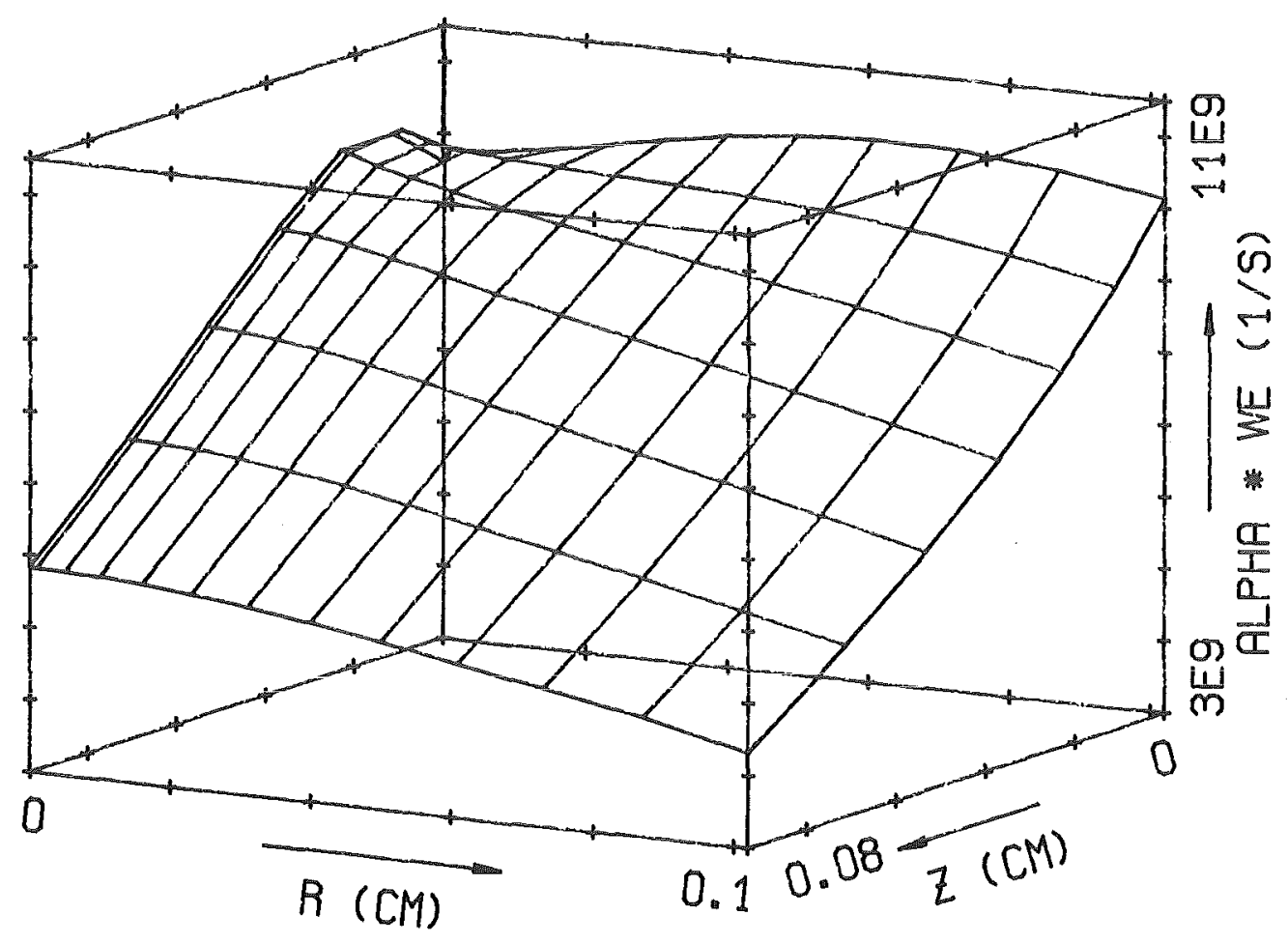

FIG. 33. IONIZATION COEFFICIENT * DRIFT VELOCITY AT T=508 NS 


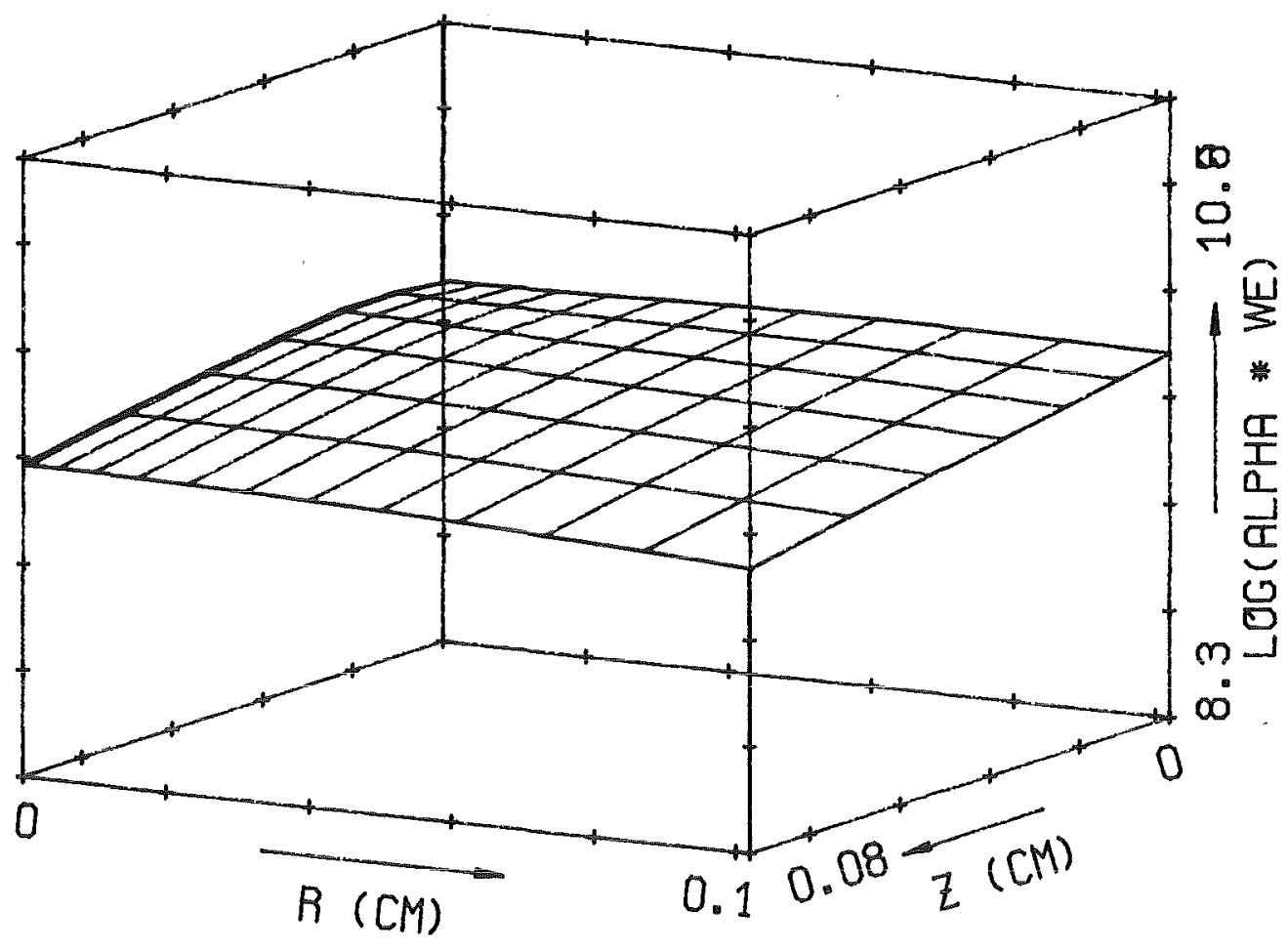

FIG. 34. LOG(IONIZATION COEFFICIENT * DRIFT VELOCITY), 508 NS

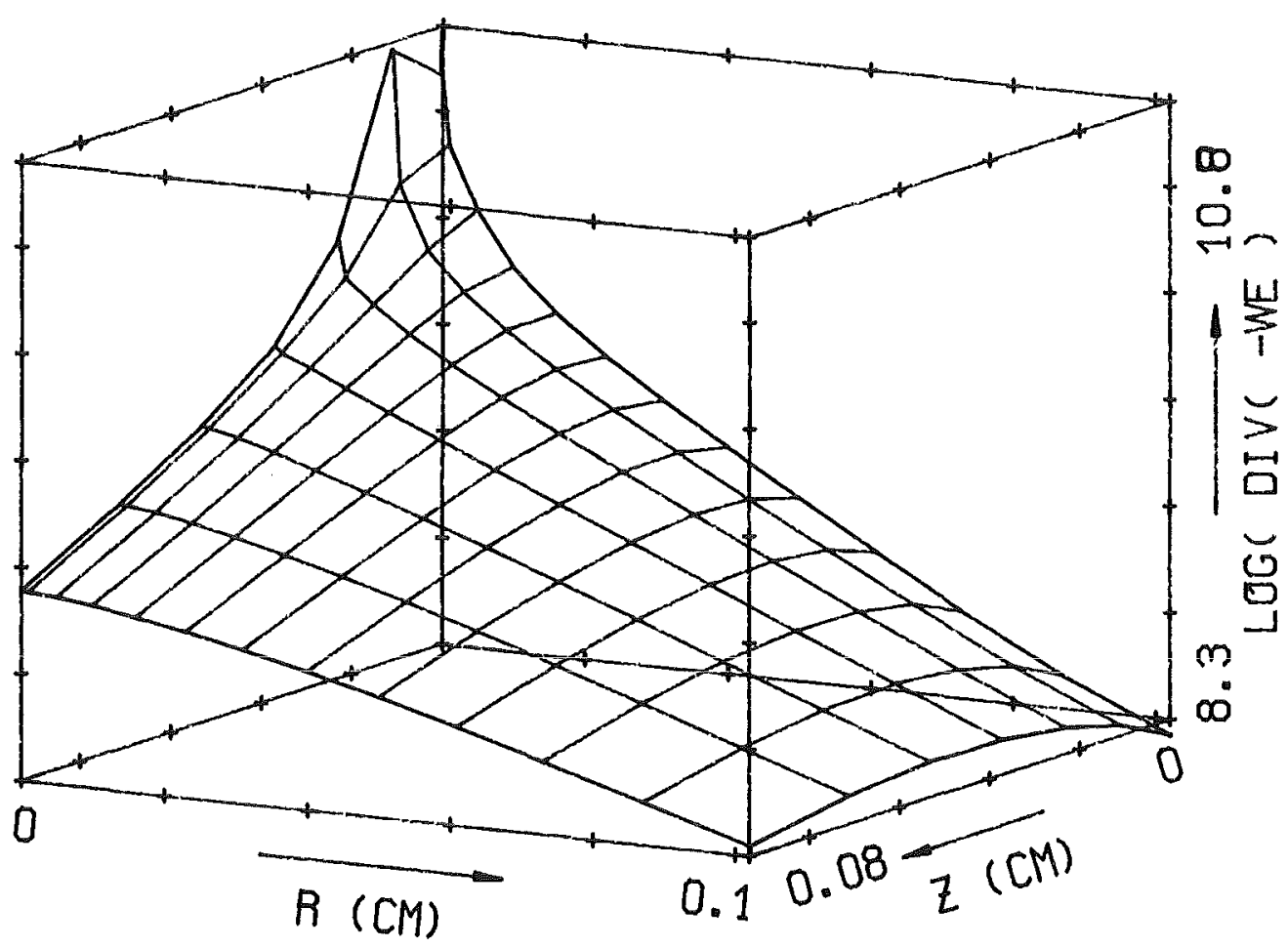

FIG. 35. LOG( DIV( - ELECTRON DRIFT VELOCITY ) ) AT T=508 NS 


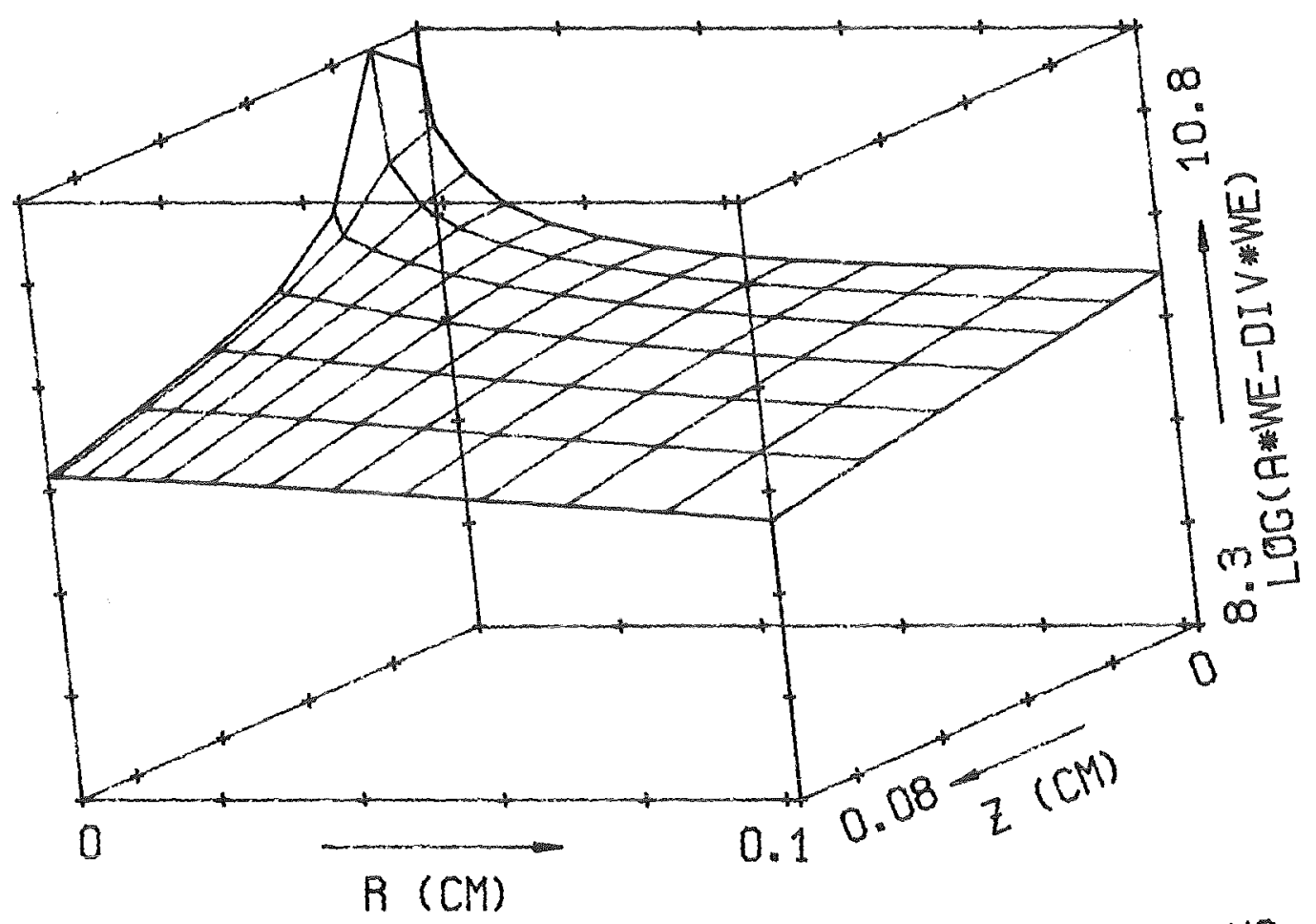

FIG 36. LOG( ALPHA * WE - DIV(WE)) AT T=508 NS 